\title{
Targeted proteomics reveals promising biomarkers of disease activity and organ involvement in antineutrophil cytoplasmic antibody-associated vasculitis
}

Jun Ishizaki ${ }^{1}$, Ayako Takemori ${ }^{2}$, Koichiro Suemori ${ }^{1}$, Takuya Matsumoto ${ }^{1}$, Yoko Akita' ${ }^{1}$, Ken-ei Sada ${ }^{3}$, Yukio Yuzawa ${ }^{4}$, Koichi Amano ${ }^{5}$, Yoshinari Takasaki ${ }^{6}$, Masayoshi Harigai ${ }^{7}$, Yoshihiro Arimura ${ }^{8}$, Hirofumi Makino ${ }^{9}$, Masaki Yasukawa ${ }^{1}$, Nobuaki Takemori ${ }^{2^{*}}$, Hitoshi Hasegawa ${ }^{1 *}$ (i) and for the Research Committee of Intractable Vasculitis Syndrome and the Research Committee of Intractable Renal Disease of the Ministry of Health, Labour and Welfare of Japan

\begin{abstract}
Background: Targeted proteomics, which involves quantitative analysis of targeted proteins using selected reaction monitoring (SRM) mass spectrometry, has emerged as a new methodology for discovery of clinical biomarkers. In this study, we used targeted serum proteomics to identify circulating biomarkers for prediction of disease activity and organ involvement in antineutrophil cytoplasmic antibody (ANCA)-associated vasculitis (AAV).

Methods: A large-scale SRM assay targeting 135 biomarker candidates was established using a triple-quadrupole mass spectrometer coupled with nanoflow liquid chromatography. Target proteins in serum samples from patients in the active and remission (6 months after treatment) stages were quantified using the established assays. Identified marker candidates were further validated by enzyme-linked immunosorbent assay using serum samples ( $n=169)$ collected in a large-cohort Japanese study (the RemIT-JAV-RPGN study).

Results: Our proteomic analysis identified the following proteins as biomarkers for discriminating patients with highly active AAV from those in remission or healthy control subjects: tenascin C (TNC), C-reactive protein (CRP), tissue inhibitor of metalloproteinase 1 (TIMP1), leucine-rich alpha-2-glycoprotein 1, S100A8/A9, CD93, matrix metalloproteinase 9, and transketolase (TKT). Of these, TIMP1 was the best-performing marker of disease activity, allowing distinction between mildly active AAV and remission. Moreover, in contrast to CRP, serum levels of TIMP1 in patients with active AAV were significantly higher than those in patients with infectious diseases. The serum levels of TKT and CD93 were higher in patients with renal involvement than in those without, and they predicted kidney outcome. The level of circulating TNC was elevated significantly in patients with lung infiltration. AAV severity was associated with markers reflecting organ involvement (TKT, CD93, and TNC) rather than inflammation. The eight markers and myeloperoxidase (MPO)-ANCA were clustered into three groups: MPO-ANCA, renal involvement (TKT and CD93), and inflammation (the other six markers).

(Continued on next page)
\end{abstract}

\footnotetext{
*Correspondence: takemori@m.ehime-u.ac.jp; hitoshih@m.ehime-u.ac.jp

${ }^{2}$ Division of Proteomics Research, Proteo-Science Center, Ehime University,

Toon, Ehime 791-0295, Japan

'Department of Hematology, Clinical Immunology, and Infectious Diseases,

Ehime University Graduate School of Medicine, Toon, Ehime 791-0295, Japan

Full list of author information is available at the end of the article
} 
(Continued from previous page)

Conclusions: We have identified promising biomarkers of disease activity, disease severity, and organ involvement in AAV with a targeted proteomics approach using serum samples obtained from a large-cohort Japanese study. Especially, our analysis demonstrated the effectiveness of TIMP1 as a marker of AAV activity. In addition, we identified TKT and CD93 as novel markers for evaluation of renal involvement and kidney outcome in AAV.

Keywords: Antineutrophil cytoplasmic antibody-associated vasculitis, Biomarkers, Targeted proteomics, Microscopic polyangiitis, Granulomatosis with polyangiitis, Eosinophilic granulomatosis with polyangiitis

\section{Background}

The antineutrophil cytoplasmic antibody (ANCA)-associated vasculitides (AAV) comprise three distinct diseases: granulomatosis with polyangiitis (GPA), microscopic polyangiitis (MPA), and eosinophilic granulomatosis with polyangiitis (EGPA) [1]. AAV are characterized by pauci-immune necrotizing inflammation of small to medium-sized vessels and affect multiple organs. ANCA have been shown to play a potential role in the pathogenesis of vasculitis [2]. However, use of ANCA for monitoring disease activity is insufficiently sensitive or specific [3-7]. Traditional acute-phase indicators, including $\mathrm{C}$-reactive protein (CRP), also lack the sensitivity and specificity for monitoring of AAV disease activity. Therefore, additional markers are needed as a guide for management and for distinguishing active disease from remission. Moreover, biomarkers that reflect organ damage are also necessary because AAV affects multiple organs. Several circulating biomarkers allowing comparison of active disease with remission in AAV have been reported in large-cohort studies and systematic literature searches [8-14]. However, these biomarkers are not yet recognized as clinically useful for monitoring of disease activity or predicting prognosis.

Targeted proteomics involves large-scale protein quantification using selected reaction monitoring (SRM; also known as multiple reaction monitoring), and its application for clinical biomarker discovery has been explored in recent years [15-24]. SRM is a quantitative mass spectrometry (MS) technique for selective detection of targeted molecules in a complex analytical sample, and it has become a broadly acceptable approach for protein quantification without the use of antibodies $[15,17,18]$. The multiple target selectivity of SRM is particularly useful for parallel monitoring of different marker proteins, enabling highly sensitive quantitation of proteins in a crude serum sample containing the proteins of interest at subfemtomolar levels. The targeted proteomics approach consists of three steps: (1) selection of novel biomarker candidates based on experimental and/ or bioinformatics information, (2) quantitative evaluation of candidate biomarkers using SRM, and (3) verification of selected candidates using an antibody-based quantitative technology such as an enzyme-linked immunosorbent assay (ELISA). This facilitates highthroughput identification of reliable biomarker candidates from limited-availability biological samples.

To analyze the characteristics of Japanese patients with AAV, the Research Committee for Intractable Vasculitis Syndrome and the Research Committee for Intractable Renal Disease of the Ministry of Health, Labour and Welfare of Japan collaboratively implemented a nationwide prospective cohort study of remission induction therapy in Japanese patients with antineutrophil cytoplasmic antibody-associated vasculitis and rapidly progressive glomerulonephritis (RemIT-JAV-RPGN) [25]. In contrast to studies $[8,9,13]$ related to the RAVE trial, which have indicated that GPA with positive proteinase-3 (PR3) is predominant in patients with AAV from the United States and The Netherlands, Japanese patients with AAV appear to have a higher incidence of MPA than of GPA, and they show a predominance of myeloperoxidase (MPO)-ANCA. In the present study, we employed targeted proteomics to identify novel circulating protein biomarkers of disease activity and severity, as well as organ damage, in AAV using serum samples obtained from a large-cohort Japanese study (RemIT-JAV-RPGN).

\section{Methods}

\section{Healthy donors and patients}

Serum samples from patients with AAV were obtained at our hospital $(n=17)$ and from a cohort study (RemIT-JAV-RPGN) $(n=152)$ [25]. All cases of AAV fulfilled the criteria for primary systemic vasculitis proposed by the European Medicines Agency (EMEA) algorithm [26]. From among 321 patients who had been enrolled initially in the RemIT-JAV-RPGN study, serum samples for use in the present study were collected from 152 patients with active disease (before treatment) and 64 patients at 6 months after the start of treatment. On the basis of the EMEA algorithm, 169 of these patients were diagnosed as follows: 105 with MPA, 36 with GPA, 25 with EGPA, and 3 with unclassifiable disease. Paired serum samples before and 6 months after the start of treatment were obtained from 79 patients: 42 with MPA, 20 with GPA, 14 with EGPA, and 3 with unclassifiable disease. 
Serum samples were also obtained from 30 healthy donors, 30 patients with active rheumatoid arthritis (RA), 21 patients with active systemic lupus erythematosus (SLE), and 25 patients with bacterial infectious diseases treated at our hospital. RA was diagnosed on the basis of the 2010 American College of Rheumatology (ACR)/ European League Against Rheumatism (EULAR) criteria for RA [27]. The criterion for the active phase of RA in this study was considered to be a Simplified Disease Activity Index $\geq 11$ [28]. Patients with SLE fulfilled the ACR revised criteria for SLE [29]. The active phase of SLE was defined as a Systemic Lupus Erythematosus Disease Activity Index score $\geq 4$, based on clinical findings in the 2 weeks prior to sample collection [30]. The 25 patients with infectious diseases included 15 with bacterial pneumonia, 4 with urinary infection, 5 with acute cholecystitis, and 1 with enterocolitis. Information on the characteristics of the healthy donors and patients is given (Table 1, Additional file 1: Table S1). Serum samples were frozen at $-80{ }^{\circ} \mathrm{C}$ until use.

\section{Outcome measures}

Details of the RemIT-JAV-RPGN study protocol were reported previously [25]. Disease activity was evaluated according to the Birmingham Vasculitis Activity Score version 3 (BVAS) system [31]. Remission was defined as complete absence of disease activity attributable to active vasculitis. Absence of disease activity was determined systematically using a BVAS of 0 on two occasions at least 1 month apart according to the EULAR recommendations [32]. Organ involvement was evaluated in accordance with the BVAS system. Renal damage was defined as a renal BVAS score $\geq 1$. Endstage renal disease (ESRD) was defined as dependence on dialysis or an irreversible increase in the serum creatinine level to $>5.6 \mathrm{mg} / \mathrm{dl}$. Lung involvement was defined as a chest BVAS score $\geq 1$.

\section{Disease severity}

The disease severity of the patients enrolled in the RemIT-JAV-RPGN study was classified as localized, early systemic, generalized, or severe in accordance with the definition of the European Vasculitis Study Group (EUVAS) [32]. Patients with threatened vital organ function were classified as having generalized disease, and patients with organ failure were classified as having severe disease. The definitions of disease severity have been described in detail previously [25].

The patients were also classified into four groups according to the Japanese rapidly progressive

Table 1 Baseline characteristics of the 169 patients with antineutrophil cytoplasmic antibody-associated vasculitis

\begin{tabular}{|c|c|c|c|c|}
\hline & All $(n=169)$ & MPA $(n=105)$ & GPA $(n=36)$ & $\mathrm{EGPA}(n=25)$ \\
\hline Male/female, $n / n$ & $65 / 104$ & $45 / 60$ & $14 / 22$ & $6 / 19$ \\
\hline Age, years & $71(61-78)$ & $73(66-78)$ & $69(60-79)$ & $60(46-70)$ \\
\hline MPO-ANCA-positive & $137(81.1 \%)$ & $103(98.1 \%)$ & $22(61.1 \%)$ & $9(36 \%)$ \\
\hline PR3-ANCA-positive & $20(12.2 \%)$ & $4(4.0 \%)$ & $15(41.7 \%)$ & $1(4.2 \%)$ \\
\hline ANCA-negative & $18(10.7 \%)$ & $1(1.0 \%)$ & $1(2.8 \%)$ & $16(64 \%)$ \\
\hline 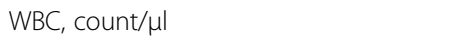 & $9400(7000-14,300)$ & $8500(6200-12,300)$ & $9950(7825-14,050)$ & $19,200(15,090-27,700)$ \\
\hline Serum creatinine, mg/dl & $0.92(0.66-2.43)$ & $1.41(0.81-3.56)$ & $0.71(0.61-1.24)$ & $0.59(0.44-0.76)$ \\
\hline eGFR, ml/minute/1.73 $\mathrm{m}^{2}$ & $50(17-74)$ & $34(12-61)$ & $65(41-75)$ & $81(68-107)$ \\
\hline \multicolumn{5}{|l|}{ Disease activity and organ involvement } \\
\hline BVAS score & $16(12-21)$ & $15(12-19)$ & $19(12-24)$ & $17(14-20)$ \\
\hline BVAS chest positive & 75 (44.4\%) & $43(41 \%)$ & $17(47.2 \%)$ & $15(60 \%)$ \\
\hline BVAS renal positive & $120(71 \%)$ & $92(87.6 \%)$ & $23(63.9 \%)$ & $4(16 \%)$ \\
\hline \multicolumn{5}{|l|}{ Disease severity } \\
\hline EUVAS disease severity, L/ES/Ge/Se, $n$ & $5 / 37 / 96 / 31$ & $2 / 27 / 52 / 24$ & $3 / 7 / 23 / 3$ & 0/1/20/14 \\
\hline RPGN clinical grading, I/II/III/IV, $n$ & $40 / 86 / 38 / 5$ & $18 / 54 / 28 / 5$ & $8 / 21 / 7 / 0$ & $14 / 9 / 2 / 0$ \\
\hline Five-Factor Score, $\leq 1 / 2 / \geq 3, n$ & $67 / 57 / 45$ & $18 / 43 / 44$ & 29/7/0 & 20/4/1 \\
\hline \multicolumn{5}{|l|}{ Outcomes at 6 months } \\
\hline Clinical remission (BVAS score $=0$ ) & 121/157 (77.1\%) & $71 / 95$ (74.7\%) & 28/35 (80\%) & 19/24 (79.2\%) \\
\hline ESRD & 15/157 (9.6\%) & $14 / 95(14.7 \%)$ & $1 / 35(2.9 \%)$ & $0 / 24(0.0 \%)$ \\
\hline
\end{tabular}

Abbreviations: eGFR Estimated glomerular filtration rate, BVAS Birmingham Vasculitis Activity Score version 3, EUVAS European Vasculitis Study Group, L/ES/Ge/Se Localized/early systemic/generalized/severe, RPGN Rapidly progressive glomerulonephritis, ESRD End-stage renal disease, MPO Myeloperoxidase, ANCA Antineutrophil cytoplasmic antibody, PR3 Proteinase-3, WBC White blood cell count, EGPA Eosinophilic granulomatosis with polyangiitis, GPA Granulomatosis with polyangiitis, MPA Microscopic polyangiitis Values are median (IQR) or $n(\%)$ 
glomerulonephritis (RPGN) clinical grading [33], which considers the following parameters: serum creatinine level $(<3 \mathrm{mg} / \mathrm{dl}=0$ points, $3-6 \mathrm{mg} / \mathrm{dl}=1$ point, $>6 \mathrm{mg} / \mathrm{dl}=2$ points, and dialysis-dependent $=3$ points), age ( $\leq 59$ years $=0,60-69$ years $=1$, and $\geq 70$ years $=2$ ), lung involvement (negative $=0$ and positive $=2$ ), and serum CRP level $(<2.6 \mathrm{mg} / \mathrm{dl}=0,2.6-10.0 \mathrm{mg} / \mathrm{dl}=1$, and $>10 \mathrm{mg} /$ $\mathrm{dl}=2$ ). Lung involvement was defined as the presence of chest symptoms in BVAS or interstitial lung disease (ILD). In the present study, ILD was diagnosed by site investigators using chest radiography and/or thoracic computed tomography. The point totals for these four parameters were summed, and patients were divided into four groups as follows: grade I, $0-2$ points; grade II, 3-5 points; grade III, $6-7$ points; and grade IV, 8-9 points.

For calculation of the Five-Factor Score (FFS) 2009 [34], renal insufficiency was defined as a serum creatinine level $>1.7 \mathrm{mg} / \mathrm{dl}$; cardiac insufficiency as the presence of cardiac symptoms in BVAS; gastrointestinal involvement as the presence of abdominal symptoms in BVAS; and ear, nose, and throat (ENT) involvement as the presence of ENT symptoms in BVAS. Age $>65$ years, cardiac insufficiency, gastrointestinal involvement, and renal insufficiency were each accorded +1 point, and absence of ENT manifestations was also accorded +1 point. The patients were divided into three groups according to the summed point totals for these five parameters: $\leq 1,2$, or $\geq 3$.

\section{Protein digestion}

Protein digestion for SRM analysis was performed as described previously [35]. Fourteen major serum proteins were depleted using a Multiple Affinity Removal Spin Cartridge Human-14 (Agilent Technologies, Santa Clara, CA, USA). For sample preparation prior to liquid chromatography-tandem mass spectrometry (LC-MS/ MS), serum proteins (5 $\mu \mathrm{g}$ of total protein) separated on NuPAGE $4-12 \%$ gel (Life Technologies, Carlsbad, CA, USA) were digested with sequencing grade trypsin (Promega, Madison, WI, USA) in $100 \mathrm{mM}$ ammonium bicarbonate $(\mathrm{pH} 8.8)$ overnight at $37^{\circ} \mathrm{C}$. Digested peptides were desalted using a self-made C18 STop And Go Extraction tip and eluted with $40 \mu \mathrm{l}$ of $0.1 \%$ ( $\mathrm{vol} / \mathrm{vol}$ ) trifluoroacetic acid (TFA)/80\% (vol/vol) acetonitrile. Eluates were dried by vacuum centrifugation and reconstituted with $10 \mu \mathrm{l}$ of $0.1 \%$ (vol/vol) TFA for MS analysis.

\section{Mass spectrometry}

MS/MS and SRM analyses were carried out on a QTRAP 5500 mass spectrometer equipped with a nanoelectrospray ionization source (SCIEX, Framingham, MA, USA) as described previously [21, 24]. Chromatographic separation of the digested peptides was performed using an Eksigent NanoLC system (SCIEX). The mobile phases consisted of $0.1 \%$ ( vol $/ \mathrm{vol})$ formic acid in $\mathrm{H}_{2} \mathrm{O}$ as solvent $\mathrm{A}$ and $0.1 \%$ (vol/vol) for$\mathrm{mic}$ acid $/ 80 \%$ (vol/vol) acetonitrile as solvent B. For MS/ MS analysis, peptide samples were injected onto a 200$\mu \mathrm{m}$ inner diameter (i.d.) $\times 0.5-\mathrm{mm}$ cHiPLC trap column (SCIEX). Concentrated peptides were then separated on a $75-\mu \mathrm{m}$ i.d. $\times 15-\mathrm{cm}$ C18 reversed-phase cHiPLC column (SCIEX) at a flow rate of $300 \mathrm{nl} /$ minute using the following gradient schedule: 0-60 minutes, $2-18 \% \mathrm{~B}$; 60-95 minutes, $18-40 \%$ B; $95-100$ minutes, $40-90 \%$ B; holding at $90 \%$ B for 5 minutes, and re-equilibration at $2 \%$ B for 15 minutes. MS/MS spectra were searched against the UniProt human proteome database using ProteinPilot software version 4.1 (SCIEX) with the following parameters: cysteine alkylation, acrylamide; digestion, trypsin; processing parameters, biological modification; and search effort, through ID.

LC-SRM assays were developed using $\left[{ }^{13} \mathrm{C}_{6},{ }^{15} \mathrm{~N}_{2}\right] \mathrm{ly}$ sine-labeled or $\left[{ }^{13} \mathrm{C}_{6},{ }^{15} \mathrm{~N}_{4}\right]$ arginine-labeled standard peptides (Sigma-Aldrich, St. Louis, MO, USA) as described previously $[21,24]$. For SRM analysis using the LC-MS system, targeted peptides were separated at a flow rate of $300 \mathrm{nl} /$ minute employing the following gradient schedule: 0-60 minutes, 2-18\% B; 60-95 minutes, $18-40 \% \mathrm{~B}$; $95-100$ minutes, $40-90 \%$ B; holding at $90 \%$ B for 5 minutes, and re-equilibration at $2 \%$ B for 15 minutes.

Quantitative analysis of the obtained SRM data was performed using Skyline software [36]. Quantification of relative protein abundance across different serum samples is based on the peak area ratios of the light (endogenous) and heavy $\left({ }^{13} \mathrm{C} /{ }^{15} \mathrm{~N}\right.$-labeled internal standard) forms of each peptide.

\section{ELISA}

Analysis of samples was performed using commercially available ELISA kits in accordance with the manufacturer's instructions. We measured heterodimer S100A8/ A9 because S100A8 and S100A9 form a heterodimer. The following ELISA kits were used: CRP, CD93, matrix metalloproteinase 9 (MMP9), S100A8/A9, and tissue inhibitor of metalloproteinase 1 (TIMP1) (R\&D Systems, Minneapolis, MN, USA); leucine-rich alpha-2glycoprotein 1 (LRG1) and tenascin C (TNC) (ImmunoBiological Laboratories, Gunma, Japan); transketolase (TKT) (LifeSpan BioSciences, Seattle, WA, USA); and MPO-ANCA (MBL, Nagoya, Japan).

\section{Statistical analysis}

Values are expressed as medians and IQRs or as numbers and percentages. Continuous nonparametric variables were compared using the Mann-Whitney $U$ test. Distinction of active AAV from remission in the same 
patients with AAV was compared using the Wilcoxon signed-rank test. Analysis of covariance was employed for comparison analysis when adjustment was necessary for age, sex, and four distinct AAV groups (MPA, GPA, EGPA, and unclassifiable disease) to calculate the adjusted means for each biomarker level. When using analysis of covariance, each biomarker level was logarithmically transformed to attain a normal distribution and then compared using Student's $t$ test and Dunnett's test. For easier reading, biomarker levels presented in the tables and figures were not transformed. ROC curves were constructed for each marker using logistic regression to further assess the ability of the biomarkers and to define the optimal cutoff point. The AUC was calculated, and positive likelihood ratios (LRs) (sensitivity/[1 - specificity]) were determined at the optimal cutoff points. Correlations between paired data were analyzed using Spearman's rank correlation. Differences at $p<0.05$ were considered to be statistically significant. When comparing 44 biomarker candidates in protocol 1 or 15 in protocol 2 identified by SRM assay between active AAV and remission, statistical significance was determined by $<0.05 / 44$, or $<0.05 / 15$ by Bonferroni correction to adjust for multiple testing. Statistical analyses were performed using JMP version 9 software (SAS Institute, Cary, NC, USA).

\section{Results}

\section{Candidate screening and assay development}

The workflow for the development of SRM assays targeting marker candidates of disease activity in AAV is shown in Fig. 1a. Initially, we selected the targeted candidates by an experiment-based approach (protocol 1) in which we conducted MS-based serum proteomic profiling of patients with AAV before and after treatment. Paired serum samples before (active) and at 6 months (remission) after treatment were obtained from six patients (one EGPA, two MPA, two GPA, and one unclassifiable). After depletion of high-abundance proteins with an immunoaffinity column, the remaining proteins were subjected to LC-MS/MS analysis (Additional file 1: Figure S1a). From among the 267 proteins identified (Additional file 2: Table S2), we selected 52 proteins as candidate markers on the basis of the following criteria: (1) those specifically observed before treatment, (2) those that showed significant differences in the number of identified peptides ( $>1.5$-fold) before and after treatment, or (3) those that were endothelium-related and present in serum (Additional file 1: Figure S1b).

To maximize the chance of discovering marker proteins in this study, we further selected 87 endotheliumrelated proteins, which, based on information in publicly available databases, were expected to be present in blood [37, 38] (Fig. 1a, protocol 2). In total, 135 proteins were selected for development of SRM assays (Additional file
1: Table S3). On the basis of their biological functions, we classified the identified proteins into four groups (Fig. 1b) involved in the following: (1) cell adhesion and extracellular matrix organization (56 proteins), (2) angiogenesis and wound healing (31 proteins), (3) inflammatory responses (22 proteins), and (4) regulation of metabolism and basic cellular processes (26 proteins).

Development of a reliable SRM assay generally requires predetermination of target peptides that are suitable for SRM quantification. For all the proteins in protocol 1, we selected the target peptides from the peptide dataset observed in our MS/MS analysis. Owing to the lack of available MS/MS information for the proteins in protocol 2, we referenced the MS-based proteomic information in public databases (PeptideAtlas [39] and Plasma Proteome Database [40]), and we selected target peptides for the assay construction. All of the peptides selected for assay development are listed in Additional file 1: Table S3. To establish LC-SRM assays targeting all of the selected peptides, we generated ${ }^{13} \mathrm{C} /{ }^{15} \mathrm{~N}$-labeled synthetic peptide analogues for use as standard reference material in SRM analysis. MS/MS data obtained from synthesized reference peptides were used to generate a spectral library for SRM assay development. On the basis of the established library, the fragment ions for each of the precursor ions were selected for the final assay (Additional file 1: Figure S2).

Although SRM has a high capability to detect targeted proteins in biological samples, it is always necessary to reduce the complexity of the sample for analysis of the minor protein components. In this study, we conducted immunoaffinity depletion of the 14 most abundant proteins to enhance the SRM detectability of proteins with lower abundance. On one hand, the sample pretreatment in our experimental workflow enabled us to detect the targeted serum proteins at a low $\mathrm{ng} / \mathrm{ml}$ level in a $10-\mu \mathrm{l}$ sample. On the other hand, SRM quantification of low-abundance proteins at the picograms per milliliter level (e.g., cytokines and chemokines in human serum) still challenges current MS capability. In addition, we excluded complement components, which show physiologically relevant interactions with immune-depleted proteins during sample pretreatment, from consideration as targeted candidates for SRM assay.

\section{Targeted proteomics of selected candidates}

Using the established SRM assay, we performed parallel monitoring of target peptides and obtained LC-SRM chromatographs of each peptide from paired serum samples (before and at 6 months after treatment of the same patients) that were prepared from 23 patients (9 MPA, GPA 7, 5 EGPA, and 2 unclassifiable) in protocol 1 and from 29 patients (10 MPA, $9 \mathrm{GPA}$, and $10 \mathrm{EGPA}$ ) in 
A

\section{Step 1: Candidate Screening}

Protocol 1: experimental screening Serum proteomic profiling by LC-MS/MS

- EGPA (1) • GPA (2)

-MPA (2) • Unclassifiable (1)

$$
\text { ए }
$$

52 candidates

- Differentially observed proteins in before treatment - Circulating endothelium-related proteins

Protocol 2: in silico screening Public database search

- GeneCards (www.genecards.org)

-The Human Protein Atlas (www.proteinatlas.org) 87 candidates

Endothelium-related proteins (Expected to be released in blood)

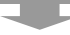

Step 2: Development of SRM Assays

Target peptide selection

Generation of ${ }^{13} \mathrm{C} /{ }^{15} \mathrm{~N}$-labeled reference peptides Optimization of SRM parameters

Step 3: Targeted Proteomic Analysis

- Protocol 1 (23 patients) • Protocol 2 (29 patients)

\section{B}

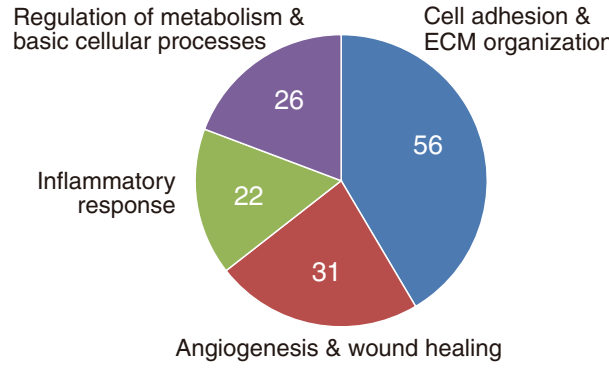

C

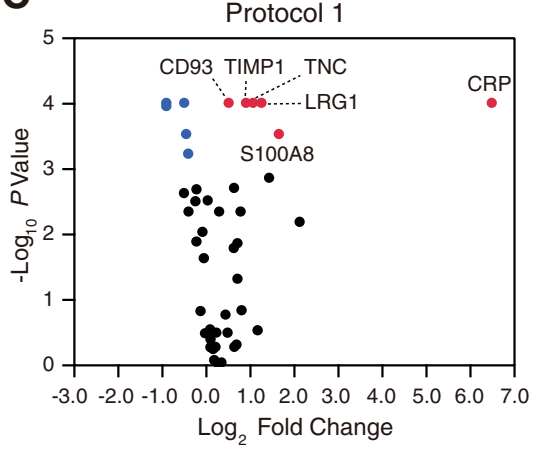

D

Protocol 2

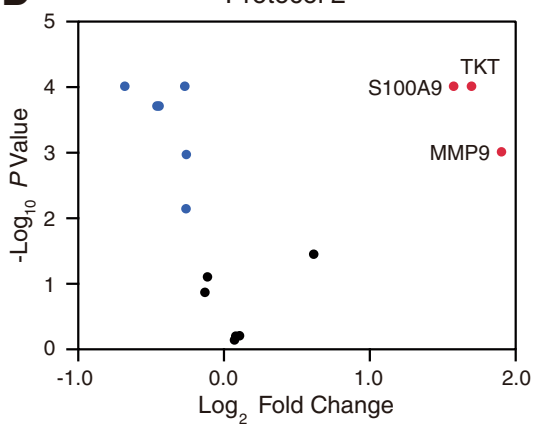

$\mathbf{E}$

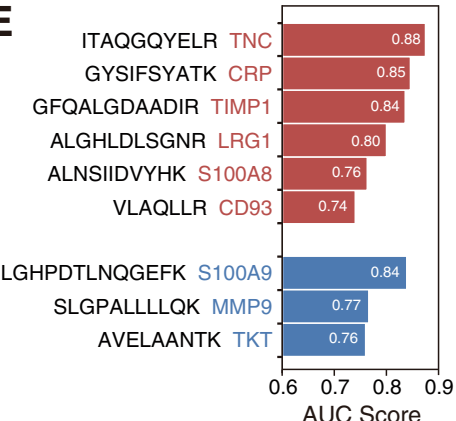

Fig. 1 Identification of candidate biomarkers of disease activity in antineutrophil cytoplasmic antibody-associated vasculitis (AAV) using the selected reaction monitoring (SRM) assay. a Workflow for the development of SRM assays targeting marker candidates of disease activity in AAV. b Functional classification of marker candidates. c Comparison of 44 candidate markers discriminating between highly active AAV (before treatment) and remission (at 6 months after the start of treatment) using the SRM assay in protocol 1. Paired serum samples prepared from 23 patients (9 microscopic polyangiitis [MPA], 7 granulomatosis with polyangiitis [GPA], 5 eosinophilic granulomatosis with polyangiitis [EGPA], and 2 unclassifiable) were used in protocol 1. d Comparison of 15 candidate markers discriminating between highly active AAV and remission using the SRM assay in protocol 2. Paired serum samples prepared from 29 patients (10 MPA, 9 GPA, and 10 EGPA) were used in protocol 2. Volcano plots are used to look at fold changes and statistical significance simultaneously. Dot plots show $-\log _{10}\left(p\right.$ values) on the ordinate and $\log _{2}$ (fold change values) on the abscissa for all activity marker candidates. The upper corners of the plot (red and blue dots) represent proteins that show both statistical significance and large fold changes. Red and blue dots indicate the proteins that were downregulated and upregulated after treatment, respectively. $p$ Values were determined by Wilcoxon signedrank test, and statistical significance was determined by $<0.05 / 44$ or $<0.05 / 15$ by Bonferroni correction. e Proteins downregulated after treatment with AUC scores $>0.7$ in protocols 1 and 2. The upper six proteins were identified in protocol 1 and the lower three proteins in protocol 2. CRP C-reactive protein, ECM Extracellular matrix, LRG1 Leucine-rich alpha-2-glycoprotein 1, MMP9 Matrix metalloproteinase 9, TIMP1 Tissue inhibitor of metalloproteinase 1, TKT Transketolase, TNC Tenascin C

protocol 2. Of the 135 marker candidates, 74 proteins were successfully quantified in this study: 44 in protocol 1 and 30 in protocol 2 (Additional file 1: Table S4). However, in protocol 2, some proteins that were quantified in only a small number of samples were impossible to analyze statistically. Therefore, to select marker candidates using statistical analysis, of the 30 marker candidates, 15 proteins that were quantified in paired serum samples from more than 20 patients were further selected. The 11 proteins in protocol 1 and 9 proteins in 
protocol 2 showed a significant change before and at 6 months after treatment (Bonferroni correction; $p<0.05 /$ 44 in protocol 1 and $p<0.05 / 15$ in protocol 2) (Fig. 1c, d; Additional file 1: Table S5). We identified downregulated proteins after treatment with AUC $>0.7$ as candidate biomarkers of disease activity. Of these proteins, the following nine were identified as candidate biomarkers of disease activity in the SRM assay: TNC, CRP, TIMP1, LRG1, S100A8, and CD93 in protocol 1; and S100A9, MMP9, and TKT in protocol 2 (Fig. 1e).

\section{Serum levels of candidate biomarkers in active AAV, remission, and healthy control subjects}

To validate the candidate biomarkers selected by SRM, we analyzed the levels of such candidates in paired AAV serum samples before (active) and at 6 months after treatment (remission), as well as in samples from healthy control subjects, using commercial ELISA kits. In the preliminary experiment, we confirmed that the ELISA titers of markers measured in this study corresponded well with the light/heavy ratio of SRM (Additional file 1: Figure S3). The results of marker measurements in samples from active AAV and remission are shown in Figs. 2 and $3 \mathrm{a}$ and Table 2. Sixty-two patients who were in remission at 6 months comprised 33 with MPA, 15 with GPA, 11 with EGPA, and 3 with unclassifiable disease. These patients had highly active AAV before treatment (total BVAS 15, range 12-21). Serum levels of CRP, TIMP1, LRG1, TNC, S100A8/A9, CD93, TKT, and MMP9 were all significantly higher in the active stage than in remission in these 62 patients. In the $48 \mathrm{MPO}-$ ANCA-positive patients, the MPO-ANCA level was also higher in active AAV than in remission. The eight markers except for MMP9 had AUC $>0.7$ for
CRP

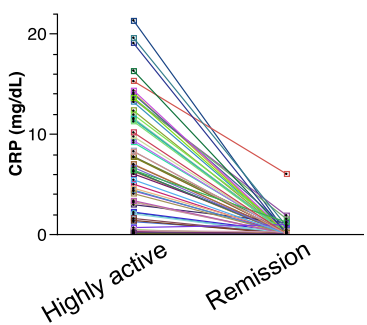

TNC

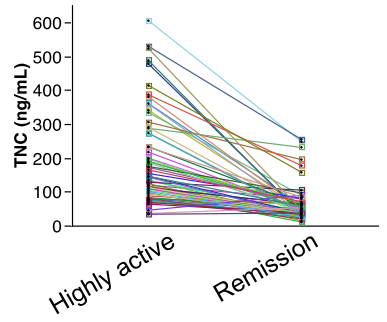

TKT

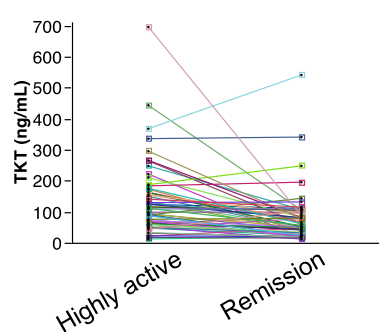

TIMP1

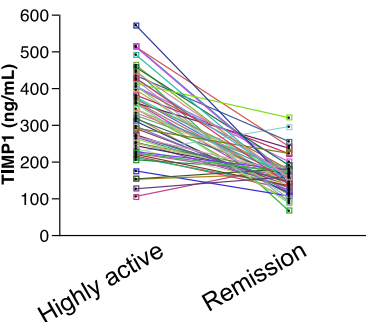

S100A8/A9

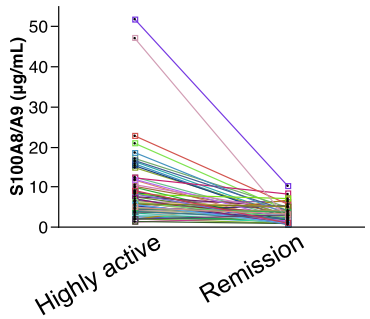

MMP9

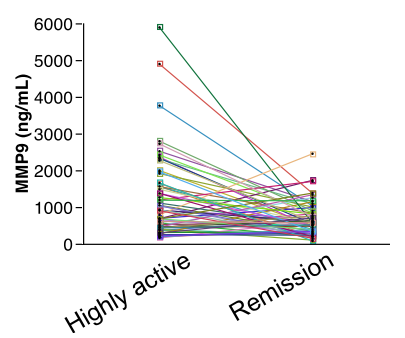

LRG1

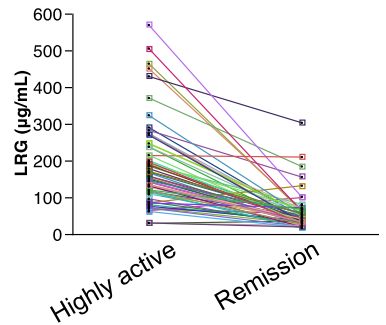

CD93

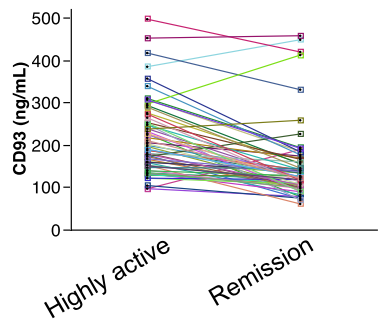

MPO-ANCA

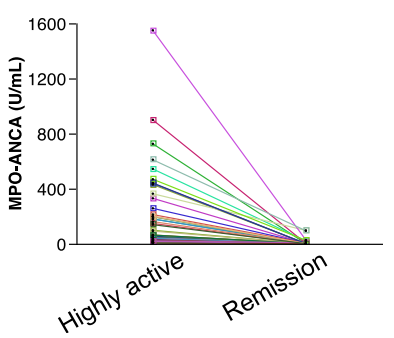

Fig. 2 Marker levels in highly active antineutrophil cytoplasmic antibody-associated vasculitis before treatment and remission at 6 months after treatment. The enzyme-linked immunosorbent assay titers of markers were measured in paired serum samples (before and at 6 months after the start of treatment) from the 62 patients with antineutrophil cytoplasmic antibody-associated vasculitis (33 microscopic polyangiitis [MPA], 15 granulomatosis with polyangiitis [GPA], 11 eosinophilic granulomatosis with polyangiitis [EGPA], and 3 unclassifiable). Each line connects the data obtained on one patient. Myeloperoxidase (MPO)-ANCA refers to only MPO-ANCA-positive patients $(n=48)$. CRP C-reactive protein, LRG1 Leucinerich alpha-2-glycoprotein 1, MMP9 Matrix metalloproteinase 9, TIMP1 Tissue inhibitor of metalloproteinase 1, TKT Transketolase, TNC Tenascin C 

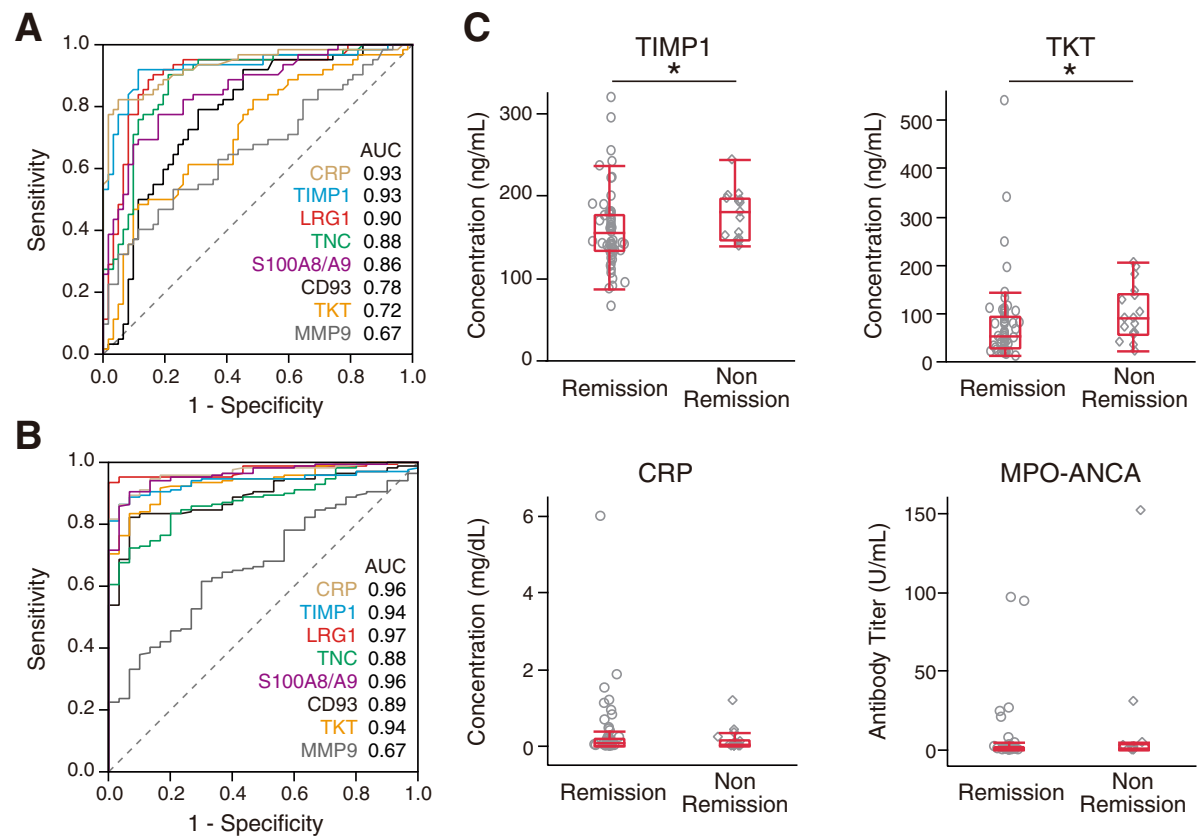

Fig. 3 a ROC curves and AUC scores showing ability of markers to distinguish highly active antineutrophil cytoplasmic antibody-associated vasculitis (AAV) from remission. The enzyme-linked immunosorbent assay (ELISA) titers of markers were measured in paired serum samples (before and at 6 months after the start of treatment) from the 62 patients with AAV. The 62 patients with highly active AAV had total Birmingham Vasculitis Activity Score version 3 (BVAS) score of 15 (range 12-21). b ROC curves and AUC scores showing ability of markers to distinguish highly active AAV from healthy control subjects. The ELISA titers of markers were compared between highly active AAV ( $n=169$, before treatment) and healthy control subjects $(n=30)$. The 169 patients before treatment had total BVAS score of 16 (range 12-21). c Comparison of marker levels between the remission group $(n=62)$ and the nonremission group $(n=17)$ at 6 months after the start of treatment. The 17 nonremission patients $(9$ microscopic polyangiitis [MPA], 5 granulomatosis with polyangiitis [GPA], and 3 eosinophilic granulomatosis with polyangiitis [EGPA]) had very mildly active AAV (total BVAS 5 , range 3-6). Each dot represents one patient. Box plots show median and IQR. Whiskers indicate most extreme points within 1.5 times the IQR of the box. $p$ Values were determined by analysis of covariance adjusted for age, sex, and four distinct AAV groups (MPA, GPA, EGPA, and unclassifiable disease). ${ }^{*} p<0.05$ (significant difference). CRP C-reactive protein, LRG1 Leucine-rich alpha-2-glycoprotein 1, MMP9 Matrix metalloproteinase 9, TIMP1 Tissue inhibitor of metalloproteinase 1, TKT Transketolase, TNC Tenascin C

Table 2 Marker levels in highly active antineutrophil cytoplasmic antibody-associated vasculitis before treatment and remission at 6 months

\begin{tabular}{|c|c|c|c|c|c|c|c|c|}
\hline Marker & Highly active $(n=62)$ & Remission $(n=62)$ & $p$ Value & $A \cup C^{a}$ & $C O P^{b}$ & Sensitivity & Specificity & $\mathrm{LR}+{ }^{c}$ \\
\hline $\mathrm{CRP}, \mathrm{mg} / \mathrm{dl}$ & $6.34(2.14-11.5)$ & $0.06(0.01-0.16)$ & $<0.0001^{d}$ & 0.93 & 1.22 & 82 & 95 & 17.0 \\
\hline TIMP1, ng/ml & $329(250-405)$ & $156(134-175)$ & $<0.0001^{d}$ & 0.93 & 205 & 92 & 89 & 8.14 \\
\hline LRG1, $\mu \mathrm{g} / \mathrm{ml}$ & $151(110-231)$ & $40.1(29.8-62.0)$ & $<0.0001^{d}$ & 0.9 & 71.9 & 89 & 85 & 6.11 \\
\hline $\mathrm{TNC}, \mathrm{ng} / \mathrm{ml}$ & $144(97.7-272)$ & $47.0(37.0-68.3)$ & $<0.0001^{d}$ & 0.88 & 72.7 & 90 & 79 & 4.31 \\
\hline $\mathrm{S} 100 \mathrm{~A} 8 / \mathrm{A} 9, \mu \mathrm{g} / \mathrm{ml}$ & $6.4(4.4-10.3)$ & $2.2(1.2-3.8)$ & $<0.0001^{d}$ & 0.86 & 4.1 & 77 & 82 & 4.36 \\
\hline $\mathrm{CD} 93, \mathrm{ng} / \mathrm{ml}$ & $192(151-252)$ & $124(103-164)$ & $<0.0001^{d}$ & 0.78 & 146 & 79 & 69 & 2.58 \\
\hline $\mathrm{TKT}, \mathrm{ng} / \mathrm{ml}$ & $104(61.8-162)$ & $52.0(28.9-91.5)$ & $<0.0001^{d}$ & 0.72 & 115 & 47 & 90 & 4.83 \\
\hline MMP9, ng/ml & $909(447-1623)$ & $566(330-839)$ & $0.0007^{d}$ & 0.67 & 897 & 53 & 77 & 2.36 \\
\hline MPO-ANCA, ${ }^{e} \mathrm{U} / \mathrm{ml}$ & $63.5(27.4-223)$ & $0.8(0.5-2.2)$ & $<0.0001^{d}$ & 0.96 & 5.1 & 98 & 88 & 7.83 \\
\hline
\end{tabular}

Abbreviations: ANCA Antineutrophil cytoplasmic antibody, COP Cutoff point, CRP C-reactive protein, $L R$ Likelihood ratio, $L R G 1$ Leucine-rich alpha-2-glycoprotein 1, MMP9 Matrix metalloproteinase 9, MPO Myeloperoxidase, TIMP1 Tissue inhibitor of metalloproteinase 1, TKT Transketolase, TNC Tenascin C

Values are median (IQR)

${ }^{\mathrm{a}} \mathrm{An}$ AUC of 1 indicates perfect discrimination between groups; an AUC of 0.5 indicates no discrimination

${ }^{\mathrm{b}}$ The maximum sum of sensitivity and specificity

'Positive likelihood ratio at the COP, which equals sensitivity/( 1 - specificity)

${ }^{\mathrm{d}} p<0.05$ by Wilcoxon signed-rank test

eOnly MPO-ANCA-positive patients $(n=48)$ 
differentiation between highly active AAV and remission. Of these, four-CRP, TIMP1, LRG1, and MPOANCA - were the best-performing markers with AUC $\geq$ 0.9 and positive LR 6.11-17.0 for distinguishing highly active AAV from remission.

A comparison of these markers between patients with active AAV and healthy control subjects is shown in Fig. $3 \mathrm{~b}$ and Table 3. Seven markers, with the exception of MMP9, also had AUC > 0.8 for distinguishing between active AAV and healthy control subjects. Of these, five-CRP, TIMP1, LRG1, S100A8/A9, and TKT-were the best-performing markers with AUC > 0.9 and positive LR 12.5-25.9 for distinguishing patients with active AAV from control subjects.

Next, we examined whether these markers were able to distinguish between nonremission (mildly active AAV) and remission at 6 months after treatment. Seventeen patients, nine with MPA, five with GPA, and three with EGPA, were in nonremission and had very mildly active AAV (total BVAS 5, range 3-6). As shown in Fig. 3c and Additional file 1: Table S6, TIMP1 and TKT were significantly higher at 6 months in the 17 nonremission patients than in the 62 remission patients. The TIMP1 cutoff point for remission was $<144 \mathrm{ng} / \mathrm{ml}$ with a sensitivity of $47 \%$ and a specificity of $94 \%$, whereas that for TKT was $<52 \mathrm{ng} / \mathrm{ml}$ with a sensitivity of $52 \%$ and a specificity of $82 \%$. There were no significant differences in the levels of TIMP1 and TKT between young and old healthy donors (Additional file 1: Table S7). In contrast, the serum levels of seven other markers-CRP, LRG1, TNC, S100A8/A9, CD93, MMP9, and MPO-ANCA - showed no significant difference between nonremission and remission at 6 months. Taken together, these findings indicated that TIMP1 was the bestperforming marker for distinguishing mildly or highly active AAV from remission or from healthy control subjects.

\section{Correlation of marker levels with AAV disease activity}

We analyzed the correlation of each marker with the total BVAS score at the active stage (before treatment). The levels of seven markers-TKT, TIMP1, S100A8/A9, CD93, TNC, CRP, and LRG1 - were correlated with total BVAS score, but the correlation was modest, with coefficients of 0.18-0.35 (Additional file 1: Figure S4). Among these markers, the strongest correlation with total BVAS score was seen for TKT (coefficient 0.35). In contrast, MPO-ANCA and MMP9 showed no correlation with the total BVAS score.

\section{Association of marker levels with organ involvement in AAV} We then examined which markers are associated with renal and lung involvement, which are often observed in AAV and affect prognosis. As shown in Fig. 4a and Additional file 1: Table S8, the serum levels of TKT and CD93 at baseline were higher in patients with active renal disease than in those without. Moreover, of the 120 patients with renal involvement, the levels of TKT and CD93 at baseline were also higher in patients who developed ESRD after 6 months than in those who did not develop ESRD (Fig. 4b). The cutoff point of TKT for development of ESRD was $>229 \mathrm{ng} / \mathrm{ml}$ with a sensitivity of $87 \%$, a specificity of $93 \%$, and an AUC of 0.91 , whereas that for CD93 was $>356 \mathrm{ng} / \mathrm{ml}$ with a sensitivity of $73 \%$, a specificity of $86 \%$, and an AUC of 0.82 . Because the levels of these markers could be affected by active renal disease and/or decreased glomerular filtration rate (GFR), the effect of GFR on the levels of these markers among patients in remission at 6 months was measured. GFR affected the levels of both TKT $(7.4 \mathrm{ng} / \mathrm{ml}$ increase per $10 \mathrm{ml} /$ minute $/ 1.73 \mathrm{~m}^{2}$ decrease in estimated glomerular filtration rate [eGFR]) and CD93 $(13.5 \mathrm{ng} / \mathrm{ml}$ increase per $10 \mathrm{ml} /$ minute $/ 1.73 \mathrm{~m}^{2}$ decrease in eGFR)

Table 3 Marker levels in patients with highly active antineutrophil cytoplasmic antibody-associated vasculitis before treatment and healthy control subjects

\begin{tabular}{|c|c|c|c|c|c|c|c|c|}
\hline Marker & Active AAV $(n=169)$ & Healthy control subjects $(n=30)$ & $p$ Value & $\mathrm{AUC}^{\mathrm{a}}$ & $\mathrm{COP}^{\mathrm{b}}$ & Sensitivity & Specificity & $\mathrm{LR}+{ }^{\mathrm{C}}$ \\
\hline $\mathrm{CRP}, \mathrm{mg} / \mathrm{dl}$ & $3.79(0.9-8.24)$ & $0.01(0.005-0.03)$ & $<0.0001^{d}$ & 0.96 & 0.16 & 86 & 97 & 25.9 \\
\hline TIMP1, ng/ml & $333(226-426)$ & $132(115-148)$ & $<0.0001^{d}$ & 0.94 & 183 & 86 & 97 & 25.9 \\
\hline $\mathrm{LRG} 1, \mu \mathrm{g} / \mathrm{ml}$ & $86.1(53.7-134)$ & $16.3(13.6-20.7)$ & $<0.0001^{d}$ & 0.97 & 28.8 & 93 & 100 & - \\
\hline $\mathrm{TNC}, \mathrm{ng} / \mathrm{ml}$ & $145(84.3-265)$ & $48.0(35.4-64.3)$ & $<0.0001^{d}$ & 0.88 & 93.1 & 72 & 93 & 10.8 \\
\hline $\mathrm{S} 100 \mathrm{~A} 8 / \mathrm{A} 9, \mu \mathrm{g} / \mathrm{ml}$ & $5.8(3.5-8.9)$ & $1.3(1.1-1.8)$ & $<0.0001^{d}$ & 0.96 & 2.2 & 91 & 93 & 13.6 \\
\hline CD93, ng/ml & $226(167-300)$ & $125(103-139)$ & $0.0005^{d}$ & 0.89 & 149 & 82 & 93 & 12.3 \\
\hline $\mathrm{TKT}, \mathrm{ng} / \mathrm{ml}$ & 124 (69.7-183) & $33.1(15.1-42.6)$ & $<0.0001^{d}$ & 0.94 & 58.1 & 83 & 93 & 12.5 \\
\hline MMP9, ng/ml & 791 (429-1382) & $517(294-838)$ & $0.0158^{d}$ & 0.67 & 642 & 62 & 70 & 2.05 \\
\hline
\end{tabular}

Abbreviations: ANCA Antineutrophil cytoplasmic antibody, COP Cutoff point, CRP C-reactive protein, LR Likelihood ratio, LRG1 Leucine-rich alpha-2-glycoprotein 1, MMP9 Matrix metalloproteinase 9, MPO Myeloperoxidase, TIMP1 Tissue inhibitor of metalloproteinase 1, TKT Transketolase, TNC Tenascin C Values are median (IQR)

${ }^{a} \mathrm{An}$ AUC of 1 indicates perfect discrimination between groups; an AUC of 0.5 indicates no discrimination

${ }^{\mathrm{b}} \mathrm{COP}$ is the maximum sum of sensitivity and specificity

${ }^{\mathrm{C}} \mathrm{LR}+$ at the COP, which equals sensitivity/(1 - specificity)

${ }^{d} p<0.05$ by analysis of covariance adjusted for age and sex 


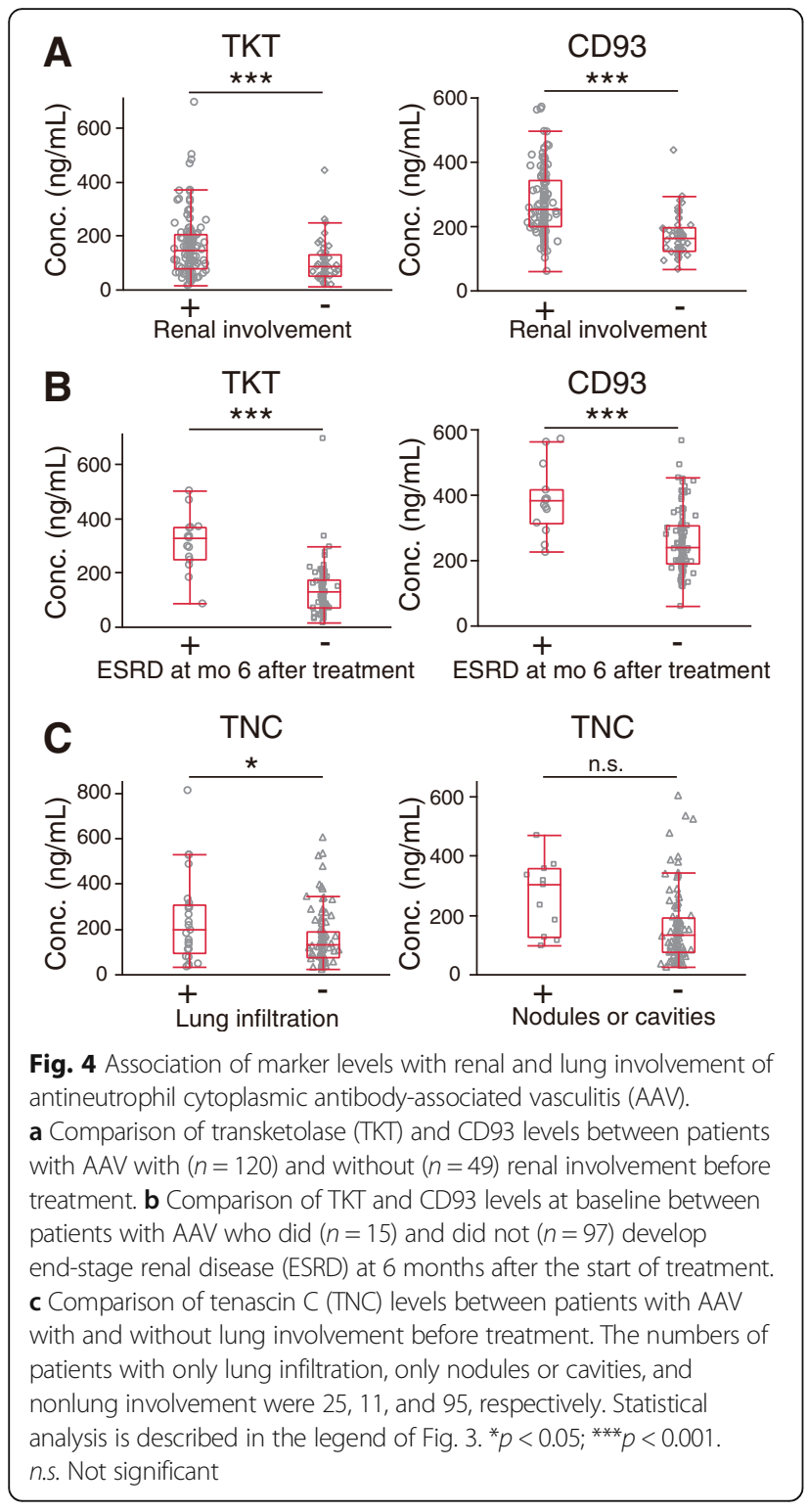

among patients in remission. Therefore, GFR appeared to be a modest contributor to the serum levels of TKT and CD93. There were no significant differences of the levels of CD93 and TKT between young and old healthy donors (Additional file 1: Table S7). Markers other than TKT and CD93 showed no significant difference between patients with and without renal disease. These findings suggested that TKT and CD93 might be excellent biomarkers for predicting kidney outcome in AAV.

We then analyzed which markers were higher in patients with AAV with lung involvement than in those without. Only the TNC level was elevated in patients with only lung infiltration, but TNC was not elevated in patients with only nodules or cavities (Fig. 4c, Additional file 1: Table S9). Associations of markers with only wheezing $(n=4)$, only endobronchial involvement $(n=0)$, only massive hemoptysis/alveolar hemorrhage $(n=3)$, and only respiratory failure $(n=0)$ could not be analyzed, owing to the small numbers of patients. Analysis of markers for pleural effusion/pleurisy $(n=14)$ was not appropriate, because the patients included those with other diseases such as heart failure and low protein. These findings suggested that TNC might act as a biomarker reflecting lung infiltration in AAV.

Comparison of marker levels among MPA, GPA, and EGPA We compared marker levels among the patients with MPA, GPA, or EGPA before treatment (Additional file 1: Table S10). There were no significant differences in the levels of CRP, TIMP1, and S100A8/A9 among the three types of AAV. The levels of CD93 and TKT were higher in MPA than in GPA or EGPA. This is because a higher proportion of patients with MPA had renal involvement in comparison with those with GPA and EGPA (88\%, 64\%, and 16\%, respectively) (Table 1$)$.

\section{Association of marker levels with disease severity in AAV}

We examined the association between marker levels and disease severity. For EUVAS-defined disease severity, serum levels of TKT and CD93 tended to be higher as severity increased. Moreover, the levels of both markers were significantly higher in patients with severe disease than in those with localized, early systemic, or generalized disease (Fig. 5a, Additional file 1: Table S11). In terms of RPGN clinical grading, the levels of six markers-TNC, CD93, TKT, TIMP1, CRP, and S100A8/A9-were significantly higher in grades III + IV patients than in grades I + II patients (Fig. 5b, c; Additional file 1: Table S11). TNC, CD93, and TKT showed AUC values of $0.79,0.76$, and 0.74 , respectively, for distinguishing between grades I + II and grades III + IV, whereas the AUC values for the other three markers-TIMP1, CRP, and S100A8/A9-were 0.68-0.73. In terms of the FFS 2009, the serum levels of CD93 and TKT were significantly higher in patients with $\geq 3$ points than in patients with $\leq 1$ or 2 points (Fig. 5d, Additional file 1: Table S11). The levels of the other six markers showed no significant differences between two groups. CD93 and TKT showed AUC values of 0.85 and 0.80 , respectively, for discrimination between two groups.

The results of analyses based on these three disease severity classification systems indicated that disease severity in patients with AAV was associated with TKT, CD93, and TNC, which are markers reflecting organ involvement, rather than with markers of inflammation such as CRP and TIMP1. 

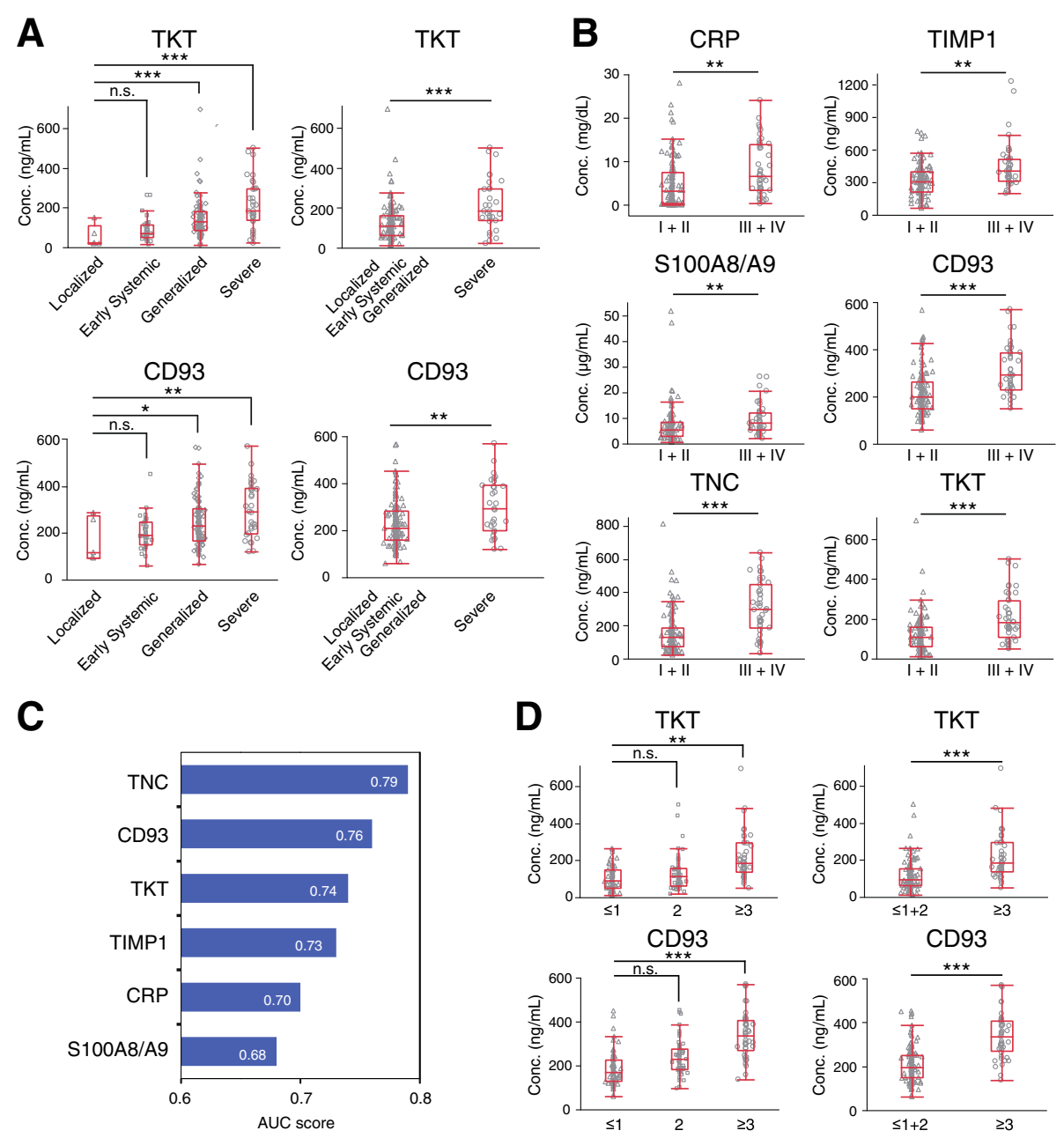

Fig. 5 Association of marker levels with disease severity in antineutrophil cytoplasmic antibody-associated vasculitis (AAV). a Association of transketolase (TKT) and CD93 levels with European Vasculitis Study Group-defined disease severity. Left panels: Comparison of the levels of each marker among the four groups: localized $(n=5)$, early systemic $(n=37)$, generalized $(n=96)$, and severe $(n=31)$ disease. Right panels: Comparison of the levels of each marker between patients with severe disease $(n=31)$ and the other three groups $(n=138)$. b Association of the level of each marker with rapidly progressive glomerulonephritis (RPGN) clinical grading. Serum levels of each marker were compared between grades I $+\|(n=126)$ and grades III + IV $(n=43)$. c AUC score for each marker distinguishing between RPGN clinical grades I + II $(n=126)$ and grades III + IV $(n=43)$. $\mathbf{d}$ Association of TKT and CD93 levels with the Five-Factor Score 2009 (FFS). Left panels: Comparison of each marker among the three groups: $\leq 1,2$, and $\geq 3$ points ( $\leq 1, n=67 ; 2$, $n=57$; and $\geq 3, n=45)$. Right panels: Comparison of the level of each marker between the patients with $\leq 1+2$ points $(n=124)$ and those with $\geq 3$ points $(n=45)$. Statistical analysis is described in the Fig. 3 legend. ${ }^{*} p<0.05 ;{ }^{* *} p<0.01 ;{ }^{* * *} p<0.001$. n.s. Not significant, CRP C-reactive protein, TIMP1 Tissue inhibitor of metalloproteinase 1, TNC Tenascin C

\section{Correlations between marker levels}

The correlations among nine markers are summarized in Fig. 6. The levels of five markers-TIMP1, LRG1, S100A8/A9, TNC, and MMP9-correlated well with the CRP value (coefficients 0.45-0.66). In contrast, MPOANCA, CD93, and TKT showed no correlation with CRP. None of the markers examined showed any correlation with MPO-ANCA (coefficients $\leq 0.22$ ). A correlation between two markers of renal involvement, TKT and CD93, was recognized (coefficient 0.55). These nine markers were clustered in the following three groups: renal involvement (CD93 and TKT), inflammation
(LRG1, CRP, MMP9, S100A8/A9, TNC, and TIMP1), and MPO-ANCA.

\section{Comparison of marker levels among AAV, RA, SLE, and infectious diseases}

We compared the serum levels of the various markers among patients with AAV, RA, SLE, and infectious diseases (Fig. 7). Patients with infectious diseases had significantly higher levels of CRP than patients with active AAV. In contrast, the serum levels of TIMP1, TKT, and CD93 were significantly higher in patients with active AAV than in those with infectious diseases. There were 


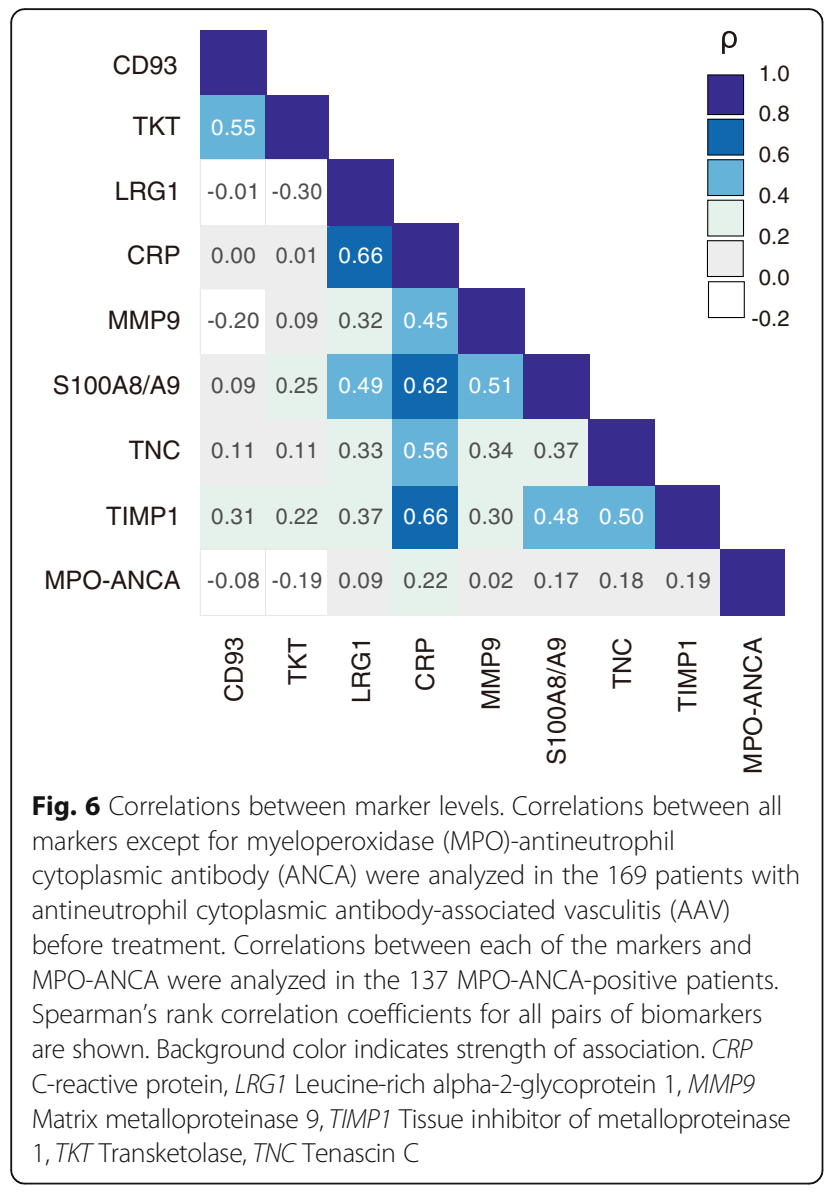

no significant differences in the levels of LRG1, TNC, S100A8/A9, and MMP9 between patients with active AAV and patients with infectious diseases. The circulating level of TKT remained low in patients with RA, SLE, and infectious diseases, although the level in the latter was slightly higher than that in healthy control subjects. These findings suggested that TKT was the bestperforming biomarker for discrimination between active AAV and other diseases such as RA, SLE, and infections.

\section{Discussion}

Targeted protein quantification using SRM has emerged as a promising new methodology for clinical biomarker discovery [15-24]. Although reliable SRM assay information on the human serum proteome still has limited public availability, multiple-target SRM quantification without the need for time-consuming antibody development is particularly useful for marker verification. In the present study, we selected 52 proteins as candidate markers for targeted proteomics on the basis of an experimental dataset derived from serum proteomic analysis. Moreover, to maximize the chance of discovering novel marker proteins, 87 vascular endothelium-related proteins were selected as targets on the basis of database search results. A total of 135 proteins were selected as potential marker candidates and subjected to targeted proteomic analysis using SRM. Of these proteins, 74 were successfully quantified. Ultimately, nine proteins-TNC, CRP, TIMP1, LRG1, CD93, S100A8, S100A9, MMP9, and TKT-were identified as candidate biomarkers of disease activity in the SRM assay.

TIMP1 is an endogenous inhibitor of MMPs and an important regulator of extracellular matrix turnover, tissue remodeling, and cellular behavior [41, 42]. TIMP1 is expressed by a variety of cell types in most human tissues. The level of circulating TIMP1 was reportedly elevated in patients with myocardial infarction, sepsis, and various cancers (reviewed in [41-44]). TIMP1 has been also described as elevated in patients with active AAV relative to those in remission or healthy control subjects $[9,45]$. Moreover, Monach et al. reported that TIMP1 allows discrimination between mild disease and remission at 6 months, although the AUC was limited at 0.68 [9]. In this study, we showed that TIMP1 was able to distinguish between patients with mildly active AAV and those in remission, whereas CRP and MPO-ANCA were unable to do so. Serum levels of TIMP1 in patients with AAV were significantly higher than those in patients with infectious diseases. In contrast, serum levels of CRP were lower in the former than in the latter. The serum levels of LRG1, MMP9, S100A8/A9, CD93, and TNC were less sensitive than that of TIMP1 for evaluation of disease activity. Our present findings and those of Monach et al. [9] suggest that TIMP1 would be superior to CRP and MPO-ANCA as a biomarker for monitoring the disease activity of AAV. Moreover, because the TIMP1 cutoff point for remission was $<144 \mathrm{ng} / \mathrm{ml}$ with a specificity of $94 \%$, this may represent the target level for achieving complete remission.

In the present study, we found two novel markers that reflected renal damage in AAV: TKT and CD93. For prediction of ESRD at 6 months, the cutoff level of TKT was $>229 \mathrm{ng} / \mathrm{ml}$ with a sensitivity of $87 \%$ and a specificity of $93 \%$, whereas that of CD93 was > $356 \mathrm{ng} / \mathrm{ml}$ with a sensitivity of $73 \%$ and a specificity of $86 \%$. Moreover, the levels of TKT and CD93 were associated with disease severity as defined by EUVAS, FFS 2009, and RPGN clinical grading. Therefore, high serum levels of TKT and CD93 are able to predict poor overall and ESRDfree survival in patients with AAV. In addition, TKT seems to be a better biomarker than CD93 for reflecting renal involvement because the level of TKT was less elevated than that of CD93 in patients with infectious diseases. TKT is a thiamine diphosphate-dependent enzyme that catalyzes several key reactions in the nonoxidative branch of the pentose phosphate pathway in the cytoplasm [46-48]. TKT is found in all mammalian tissues, and it is highly expressed in cornea, erythrocytes, 


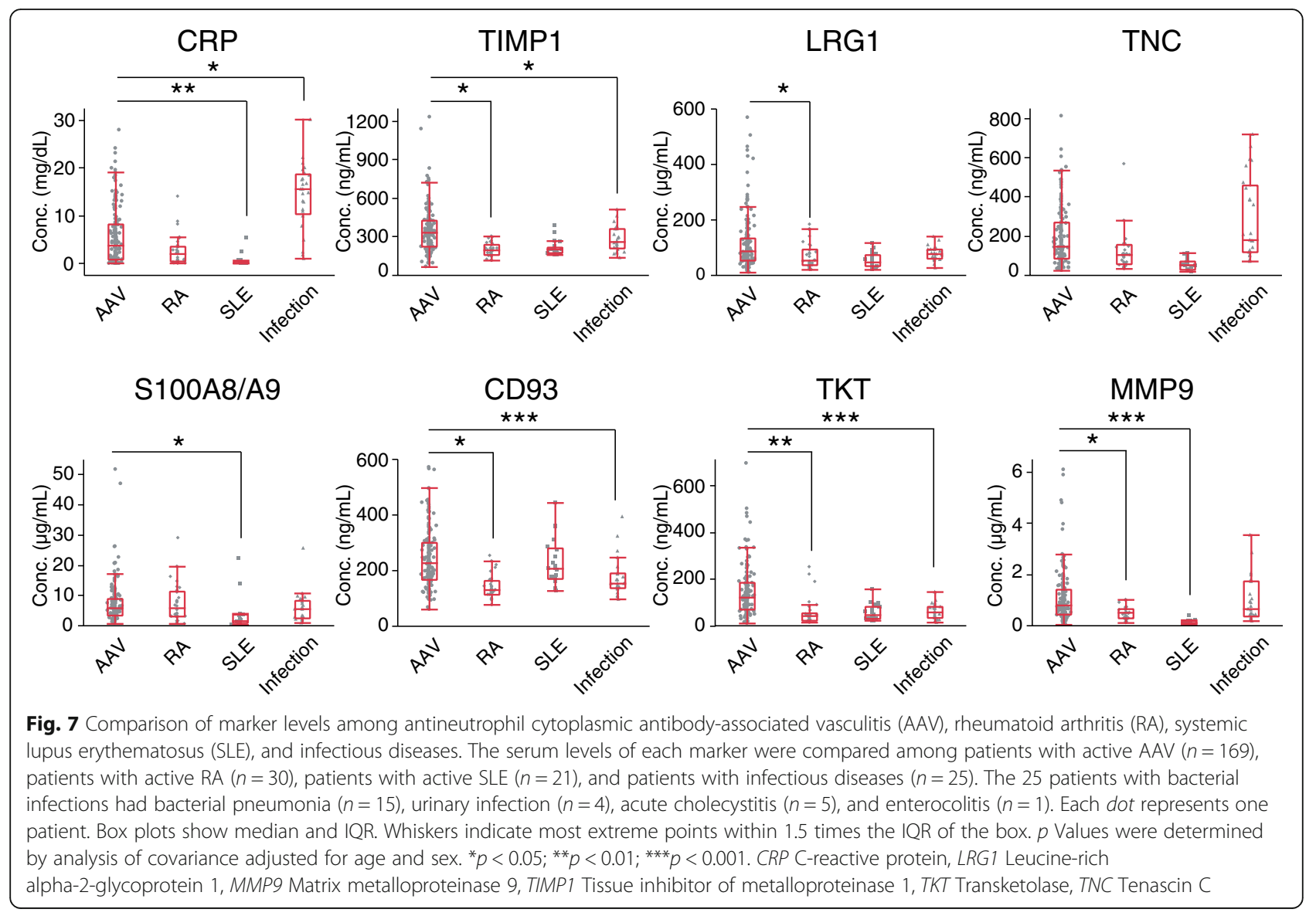

kidney, and liver [47, 49]. Because TKT is not secreted from cells, its release is a result of cell damage. Therefore, the serum level of TKT in active AAV may be associated with the degree of tissue damage. In addition, the elevated level of circulating TKT in patients with AAV with renal involvement may be due to its high expression in kidney.

CD93 is a type I transmembrane glycoprotein that is upregulated on activated neutrophils, monocytes, and vascular endothelial cells in response to inflammatory mediators such as lipopolysaccharide and tumor necrosis factor- $\alpha$, and it is shed in soluble form [50, 51]. CD93 is reportedly associated with the risk of coronary artery disease $[52,53]$. In the kidney, CD93 is expressed predominantly on infiltrating neutrophils and monocytes, as well as on interstitial and glomerular capillary endothelium [54]. The expression pattern of CD93 can explain the elevation of serum CD93 levels in our study and suggests its involvement in renal inflammation in active AAV.

It has been reported that S100A8/A9 is highly expressed within crescents and in areas of endocapillary proliferation in the kidneys of patients with AAV [55]. However, in the present study, there was no significant difference in the level of circulating S100A8/A9 in patients with or without renal involvement. S100A8/A9 is highly expressed by neutrophils, monocytes, activated macrophages, and microvascular endothelial cells, and it acts as a critical alarmin modulating the inflammatory response after its release from these cells [56]. Therefore, it is suggested that the serum S100A8/A9 level reflects the degree of inflammation more strongly than the degree of renal damage.

The extracellular matrix molecule TNC is highly expressed during embryonic development and tissue repair [57]. TNC expression has been particularly well documented in inflammatory lung conditions such as ILD, bronchial asthma, and tuberculosis $[58,59]$. In this study, we found that the serum TNC level reflected the lung infiltration in AAV and was associated with disease severity in terms of the RPGN clinical grading.

Several serum biomarkers that can distinguish between active and inactive AAV and reflect the degree of renal involvement have been reported in largecohort studies [8-13]. Monach et al. tested 28 markers of inflammation, angiogenesis, and tissue damage and repair in patients enrolled in the RAVE trial, before and 6 months after the start of treatment 
to distinguish active disease from remission [9]. They identified three promising biomarkers-MMP3, TIMP1, and CXCL13-that best discriminated these two conditions. Pepper et al. showed that the level of S100A8/A9 predicted relapse in patients with PR3ANCA treated with rituximab in the same trial [13]. Gou et al. reported that plasma levels of C3a, C5a, soluble C5b-9, and $\mathrm{Bb}$ were increased in patients with active AAV relative to those in remission [10]. Among serum biomarkers of renal involvement in AAV, the levels of MMP3 and thrombomodulin have been reported to be higher in patients with active renal disease than in those without [8]. Villacorta et al. showed that the baseline serum C3 level had prognostic value for predicting long-term renal and global survival in patients with active renal disease [12]. Brix et al. reported that CCL18 could serve as a biomarker of disease activity and renal relapse in ANCA-associated crescentic glomerulonephritis [11]. Although complement components and several lowcontent proteins such as cytokines and chemokines (present at the picograms per milliliter level in serum) have been selected as potential candidates in other studies, we eliminated them in the present study through sample pretreatment and the sensitivity limit of the SRM assay. Therefore, it may be possible to evaluate more accurately disease activity and severity, as well as organ damage, in patients with AAV by comparison among the biomarkers identified in the present and previous studies or by creating panels with such biomarkers.

\section{Conclusions}

Targeted proteomics has emerged as a promising new methodology for clinical biomarker discovery. In the present study, we identified promising biomarkers of disease activity and severity, as well as organ involvement, in AAV with a targeted proteomics approach using serum samples collected in a large-cohort Japanese study (RemIT-JAV-RPGN study). Nine proteins-CRP, TIMP1, LRG1, TNC, S100A8/A9, MMP9, CD93, TKT, and MPO-ANCA-were identified as biomarkers of disease activity. Of these, TIMP1 was the best-performing biomarker of disease activity. Moreover, we identified TKT and CD93 as biomarkers for the evaluation of renal involvement and kidney outcome, as well as TNC as a biomarker reflecting lung infiltration in AAV. AAV severity was associated with the levels of TKT, CD93, and TNC (markers reflecting organ involvement) rather than with inflammatory markers. It is expected that these biomarkers will be clinically useful for therapeutic decision-making, monitoring of disease activity, and predicting prognosis.

\section{Additional files}

Additional file 1: Figure S1. Screening of marker candidates in serum samples from patients with AAV. Figure S2. Schematic diagram of targeted proteomics using the LC-SRM assay. Figure S3. Correlations between the light/heavy ratio of SRM and the ELISA titers of markers. Figure S4 Correlation of marker levels with total BVAS score of patients with AAV. Table S1. Clinical data on healthy donors and patients with RA, SLE, and infectious diseases. Table S3. Selected marker candidates for SRM assay development. Table S4. A set of established SRM transitions for the detection of marker candidates. Table S5. The 20 proteins with a significant change before and at 6 months after treatment in protocol $1(n=23)$ and protocol $2(n=29)$ in the SRM assay. Table S6. Comparison of serum levels of biomarker candidates between the remission group $(n=62)$ and the nonremission group $(n=17)$ at 6 months after treatment. Table S7. Marker levels in old healthy donors. Table S8. Comparison of serum levels of biomarker candidates between the patients with AAV with $(n=120)$ and without $(n=49)$ renal involvement before treatment. Table S9. Comparison of serum levels of biomarker candidates between the patients with AAV with and without lung involvement before treatment. Table S10. Comparison of marker levels among MPA, GPA, and EGPA before treatment. Table S11 Association of marker levels with disease severity in patients with AAV before treatment. (PDF $2221 \mathrm{~kb}$ )

Additional file 2: Table S2. Two hundred sixty-seven proteins identified by LC-MS/MS analysis. (XLSX $106 \mathrm{~kb}$ )

\section{Abbreviations}

AAV: Antineutrophil cytoplasmic antibody-associated vasculitis; ACR: American College of Rheumatology; ANCA: Antineutrophil cytoplasmic antibody; BVAS: Birmingham Vasculitis Activity Score version 3; CRP: Creactive protein; ECM: Extracellular matrix; eGFR: Estimated glomerular filtration rate; EGPA: Eosinophilic granulomatosis with polyangiitis; ELISA: Enzyme-linked immunosorbent assay; EMEA: European Medicines Agency; ENT: Ear, nose, and throat; ESRD: End-stage renal disease; EULAR: European League Against Rheumatism; EUVAS: European Vasculitis Study Group; FFS: Five-Factor Score; GFR: Glomerular filtration rate; GPA: Granulomatosis with polyangiitis; ID: Inner diameter; ILD: Interstitial lung disease; L/ES/Ge/Se: Localized/Early systemic/Generalized/Severe; LC-MS/ MS: liquid chromatography-tandem mass spectrometry; LR: Likelihood ratio; LRG1: Leucine-rich alpha-2-glycoprotein 1; MMP9: Matrix metalloproteinase 9; MPA: Microscopic polyangiitis; MPO: Myeloperoxidase; MS: Mass spectrometry; MS/MS: Tandem mass spectrometry; n.s.: Not significant; PR3: Proteinase-3; RA: Rheumatoid arthritis; RemIT-JAV-RPGN: Cohort study of remission induction therapy in Japanese patients with antineutrophil cytoplasmic antibody-associated vasculitis and rapidly progressive glomerulonephritis; RPGN: rapidly progressive glomerulonephritis; SLE: Systemic lupus erythematosus; SRM: Selected reaction monitoring; TFA: Trifluoroacetic acid; TIMP1: Tissue inhibitor of metalloproteinase 1; TKT: Transketolase;

TNC: Tenascin C; WBC: White blood cell count

\section{Acknowledgements}

We appreciate Dr. Keiko Tanaka (Department of Epidemiology and Preventive Medicine, Ehime University Graduate School of Medicine) for help with statistical analysis and Dr. Seiichi Matsuo (Department of Nephrology, Nagoya University Hospital) for support with the experimental design. Members of the Research Committee of Intractable Vasculitis Syndrome and the Research Committee of Intractable Renal Disease of the Ministry of Health, Labour and Welfare of Japan: In addition to the authors, the following investigators and institutions participated in this study: Department of Rheumatology, Shimane University Faculty of Medicine (Yohko Murakawa); Center for Nephrology and Urology, Division of Nephrology and Dialysis, Kitano Hospital, Tazuke Kofukai Medical Research Institute (Eri Muso); Department of Hematology, Oncology, Nephrology, and Rheumatology, Akita University School of Medicine (Atsushi Komatsuda); Niigata Rheumatic Center (Satoshi Ito); Department of the Control for Rheumatic Diseases, Graduate School of Medicine, Kyoto University (Takao Fujii); Department of Immunology and Rheumatology, Clinical Neuroscience and Neurology, Endocrinology and Metabolism, Nagasaki University Graduate School of Biomedical Sciences (Atsushi Kawakami); Department of Nephrology, Iwate Prefectural Central Hospital (Izaya Nakaya); Division of Nephrology and 
Rheumatology, Department of Internal Medicine, Fukuoka University School of Medicine (Takao Saito); Shimane University, Faculty of Medicine, Division of Nephrology (Takafumi Ito); Department of Hemodialysis and Apheresis, Yokohama City University Medical Center (Nobuhito Hirawa); Center for Rheumatology, Okayama Saiseikai General Hospital (Masahiro Yamamura); Department of Medical Technology, School of Health Sciences, Faculty of Medicine, Niigata University (Masaaki Nakano); Department of Medicine, Kidney Center, Tokyo Women's Medical University (Kosaku Nitta); Division of Nephrology and Hypertension, Kashiwa Hospital, Jikei University (Makoto Ogura); Department of Nephrology, Nagoya City University Graduate School of Medical Sciences (Taio Naniwa); Division of Rheumatology and Allergology, Department of Internal Medicine, St. Marianna University School of Medicine (Shoichi Ozaki); Department of Nephrology and Endocrinology, Graduate School of Medicine, The University of Tokyo (Junichi Hirahashi); Third Department of Internal Medicine, Division of Immunology and Rheumatology, Hamamatsu University School of Medicine, Hamamatsu (Noriyoshi Ogawa); Division of Kidney and Hypertension, Department of Internal Medicine, Jikei University School of Medicine (Tatsuo Hosoya); Division of Nephrology, Department of Laboratory Medicine, Institute of Medical, Pharmaceutical and Health Sciences, Faculty of Medicine, Kanazawa University (Takashi Wada); Division of Nephrology, Department of Internal Medicine, Juntendo University Faculty of Medicine (Satoshi Horikoshi); Institute of Rheumatology, Tokyo Women's Medical University (Yasushi Kawaguchi); Division of Clinical Immunology, Graduate School of Comprehensive Human Sciences, University of Tsukuba (Taichi Hayashi); Department of Nephrology, Tokyo Medical University Hachioji Medical Center (Masaharu Yoshida); Department of Nephrology, Hypertension, Diabetology, Endocrinology and Metabolic, Fukushima Medical University (Tsuyoshi Watanabe); Department of Nephrology, Japanese Red Cross Nagoya Daini Hospital (Daijo Inaguma); Department of Integrated Therapy for Chronic Kidney Disease, Kyushu University (Kazuhiko Tsuruya); Niigata Prefectural Shibata Hospital (Noriyuki Homma); Division of Rheumatology, Department of Internal Medicine, Keio University School of Medicine (Tsutomu Takeuchi); Cardiovascular Respiratory and Neurology Division, Department of Internal Medicine, Asahikawa Medical University (Naoki Nakagawa); Kurobe City Hospital (Shinichi Takeda); Fukuoka Higashi Medical Center (Ritsuko Katabuchi); Division of Nephrology, Department of Medicine, Faculty of Medical Sciences, University of Fukui (Masayuki Iwano); Division of Rheumatology, Endocrinology and Nephrology at the Graduate School of Medicine, Hokkaido University (Tatsuya Atsumi); Department of Hemovascular Medicine and Artificial Organs, Faculty of Medicine, Miyazaki University (Shoichi Fujimoto); Division of Rheumatology and Nephrology, Department of Internal Medicine, Aichi Medical School of Medicine (Shogo Banno); Tokyo Metropolitan Geriatric Hospital and Institute of Gerontology (Takahiko Sugihara); Department of Nephrology, Tokyo Medical University Ibaraki Medical Center (Masaki Kobayashi); Department of Nephrology, Faculty of Medicine, University of Tsukuba (Kunihiro Yamagata); Department of Respiratory Medicine, Toho University Omori Medical Center (Sakae Homma); Division of Endocrinology and Metabolism, Hematology, Rheumatology and Respiratory Medicine, Department of Internal Medicine, Faculty of Medicine, Kagawa University (Hiroaki Dobashi); Department of Nephrology, Nagoya University Hospital (Naotake Tsuboi); Faculty of Health Sciences, Hokkaido University (Akihiro Ishizu); Department of Chronic Kidney Disease and Peritoneal Dialysis, Okayama University Graduate School of Medicine (Hitoshi Sugiyama).

\section{Funding}

This study was supported by a grant (XA49003) from Ehime University (to $\mathrm{HH}$ and NT), as well as by grants from the Research Committee of Intractable Vasculitis Syndrome and the Research Committee of Intractable Renal Disease of the Ministry of Health, Labour and Welfare of Japan and the study group for strategic exploration of drug seeds for ANCA-associated vasculitis and construction of clinical evidence from the Japan Agency for Medical Research and Development (AMED).

\section{Availability of data and materials}

All data generated or analyzed during this study are included in this published article and its supplementary information files.

\section{Authors' contributions}

$\mathrm{HH}$ designed the study, collected the samples and clinical data, conducted the laboratory experiments, analyzed data, and drafted manuscript. NT designed the study, examined and interpreted the proteomics, analyzed data, and drafted manuscript. JI conducted the laboratory experiments, performed statistical analyses, analyzed data, and drafted manuscript. AT examined and interpreted the proteomics. KSu, TM, and YAk conducted the laboratory experiments. KSa, YY, KA, YT, MH, and YAr provided human patient samples and clinical data. MY, KSa, YY, KA, YT, MH, YAr, and HM edited the manuscript. All authors read and approved the final manuscript.

\section{Ethics approval and consent to participate}

Approval for this study was obtained from the institutional review board of Ehime University Hospital (approval number 1109012). Written informed consent was obtained in accordance with the Declaration of Helsinki.

\section{Consent for publication}

Not applicable.

\section{Competing interests}

$\mathrm{MH}$ has received research grants and/or honoraria from Abbott Japan Co., Ltd.; Astellas Pharma Inc;; Bristol-Myers Squibb K.K.; Chugai Pharmaceutical Co., Ltd.; Eisai Co., Ltd.; Janssen Pharmaceutical K.K.; Mitsubishi Tanabe Pharma Co.; Santen Pharmaceutical Co.; Ltd.; Takeda Pharmaceutical Co., Ltd.; Teijin Pharma, Ltd.; and Pfizer Japan Inc. HM is a consultant for AbbVie and Teijin Pharma, Ltd.. The other authors declare that they have no competing interests.

\section{Publisher's Note}

Springer Nature remains neutral with regard to jurisdictional claims in published maps and institutional affiliations.

\section{Author details}

${ }^{1}$ Department of Hematology, Clinical Immunology, and Infectious Diseases, Ehime University Graduate School of Medicine, Toon, Ehime 791-0295, Japan. ${ }^{2}$ Division of Proteomics Research, Proteo-Science Center, Ehime University, Toon, Ehime 791-0295, Japan. ${ }^{3}$ Department of Nephrology, Rheumatology, Endocrinology and Metabolism, Okayama University Graduate School of Medicine, Dentistry and Pharmaceutical Sciences, Okayama, Japan. ${ }^{4}$ Department of Nephrology, Fujita Health University School of Medicine, Aichi, Japan. ${ }^{5}$ Department of Rheumatology and Clinical Immunology, Saitama Medical Center, Saitama Medical University, Saitama, Japan. ${ }^{6}$ Department of Rheumatology, Juntendo University Koshigaya Hospital, Saitama, Japan. ${ }^{7}$ Division of Epidemiology and Pharmacoepidemiology of Rheumatic Diseases, Institute of Rheumatology, Tokyo Women's Medical University, Tokyo, Japan. ${ }^{8}$ Nephrology and Rheumatology, First Department of Internal Medicine, Kyorin University School of Medicine, Tokyo, Japan.

${ }^{9}$ Okayama University Hospital, Okayama, Japan.

Received: 15 May 2017 Accepted: 15 September 2017

Published online: 29 September 2017

\section{References}

1. Jennette JC, Falk RJ, Bacon PA, Basu N, Cid MC, Ferrario F, et al. 2012 Revised International Chapel Hill Consensus Conference Nomenclature of Vasculitides. Arthritis Rheum. 2013;65(1):1-11.

2. Xiao H, Hu P, Falk RJ, Jennette JC. Overview of the pathogenesis of ANCAassociated vasculitis. Kidney Dis (Basel). 2016;1(4):205-15.

3. Falk RJ, Hoffman GS. Controversies in small vessel vasculitis - comparing the rheumatology and nephrology views. Curr Opin Rheumatol. 2007;19(1):1-9.

4. Boomsma MM, Stegeman CA, van der Leij MJ, Oost W, Hermans J, Kallenberg CG, et al. Prediction of relapses in Wegener's granulomatosis by measurement of antineutrophil cytoplasmic antibody levels: a prospective study. Arthritis Rheum. 2000;43(9):2025-33.

5. Girard T, Mahr A, Noël LH, Cordier JF, Lesavre P, André MH, et al. Are antineutrophil cytoplasmic antibodies a marker predictive of relapse in Wegener's granulomatosis? A prospective study. Rheumatology (Oxford). 2001:40(2):147-51.

6. Birck R, Schmitt WH, Kaelsch IA, van der Woude FJ. Serial ANCA determinations for monitoring disease activity in patients with ANCAassociated vasculitis: systematic review. Am J Kidney Dis. 2006;47(1):15-23. 
7. Tomasson G, Grayson PC, Mahr AD, Lavalley M, Merkel PA. Value of ANCA measurements during remission to predict a relapse of ANCA-associated vasculitis - a meta-analysis. Rheumatology (Oxford). 2012;51(1):100-9.

8. Monach PA, Tomasson G, Specks U, Stone JH, Cuthbertson D, Krischer J, et al. Circulating markers of vascular injury and angiogenesis in antineutrophil cytoplasmic antibody-associated vasculitis. Arthritis Rheum. 2011;63(12): 3988-97.

9. Monach PA, Warner RL, Tomasson G, Specks U, Stone JH, Ding L, et al. Serum proteins reflecting inflammation, injury and repair as biomarkers of disease activity in ANCA-associated vasculitis. Ann Rheum Dis. 2013;72(8): 1342-50.

10. Gou SJ, Yuan J, Chen M, Yu F, Zhao MH. Circulating complement activation in patients with anti-neutrophil cytoplasmic antibody-associated vasculitis. Kidney Int. 2013;83(1):129-37

11. Brix SR, Stege G, Disteldorf E, Hoxha E, Krebs C, Krohn S, et al. CC chemokine ligand 18 in ANCA-associated crescentic GN. J Am Soc Nephrol. 2015;26(9):2105-17.

12. Villacorta J, Diaz-Crespo F, Acevedo M, Cavero T, Guerrero C, Praga M, et al. Circulating C3 levels predict renal and global outcome in patients with renal vasculitis. Clin Rheumatol. 2016;35(11):2733-40.

13. Pepper RJ, Draibe JB, Caplin B, Fervenza FC, Hoffman GS, Kallenberg CG, et al. The association of serum calprotectin (S100A8/S100A9) levels with disease relapses in PR3-ANCA-associated vasculitis. Arthritis Rheumatol. 2016;69(1):185-93.

14. Kronbichler A, Kerschbaum J, Gründlinger G, Leierer J, Mayer G, Rudnicki M. Evaluation and validation of biomarkers in granulomatosis with polyangiitis and microscopic polyangiitis. Nephrol Dial Transplant. 2016;31(6):930-6.

15. Picotti $P$, Aebersold R. Selected reaction monitoring-based proteomics: workflows, potential, pitfalls and future directions. Nat Methods. 2012;9(6): 555-66.

16. Hüttenhain R, Soste M, Selevsek N, Röst H, Sethi A, Carapito C, 142, et al. Reproducible quantification of cancer-associated proteins in body fluids using targeted proteomics. Sci Transl Med. 2012;4:142ra94.

17. Gillette MA, Carr SA. Quantitative analysis of peptides and proteins in biomedicine by targeted mass spectrometry. Nat Methods. 2013;10(1):28-34.

18. Kennedy JJ, Abbatiello SE, Kim K, Yan P, Whiteaker JR, Lin C, et al. Demonstrating the feasibility of large-scale development of standardized assays to quantify human proteins. Nat Methods. 2014;11(2):149-55.

19. Surinova $S$, Choi M, Tao S, Schüffler PJ, Chang $C Y$, Clough $T$, et al. Prediction of colorectal cancer diagnosis based on circulating plasma proteins. EMBO Mol Med. 2015;7(9):1166-78.

20. Surinova S, Radová L, Choi M, Srovnal J, Brenner H, Vitek O, et al. Noninvasive prognostic protein biomarker signatures associated with colorectal cancer. EMBO Mol Med. 2015;7(9):1153-65.

21. Takemori N, Takemori A, Matsuoka K, Morishita R, Matsushita N, Aoshima M, et al. High-throughput synthesis of stable isotope-labeled transmembrane proteins for targeted transmembrane proteomics using a wheat germ cellfree protein synthesis system. Mol Biosyst. 2015;11(2):361-5.

22. Kim Y, Jeon J, Mejia S, Yao CQ, Ignatchenko V, Nyalwidhe JO, et al. Targeted proteomics identifies liquid-biopsy signatures for extracapsular prostate cancer. Nat Commun. 2016;7:11906

23. Kusebauch U, Campbell DS, Deutsch EW, Chu CS, Spicer DA, Brusniak MY, et al. Human SRMAtlas: a resource of targeted assays to quantify the complete human proteome. Cell. 2016;166(3):766-78.

24. Takemori N, Takemori A, Tanaka Y, Ishizaki J, Hasegawa H, Shiraishi A, et al. High-throughput production of a stable isotope-labeled peptide library for targeted proteomics using a wheat germ cell-free synthesis system. Mol Biosyst. 2016;12(8):2389-93.

25. Sada KE, Harigai M, Amano K, Atsumi T, Fujimoto S, Yuzawa Y, et al. Comparison of severity classification in Japanese patients with antineutrophil cytoplasmic antibody-associated vasculitis in a nationwide, prospective, inception cohort study. Mod Rheumatol. 2016;26(5):730-7.

26. Watts R, Lane S, Hanslik T, Hauser T, Hellmich B, Koldingsnes W, et al. Development and validation of a consensus methodology for the classification of the ANCA-associated vasculitides and polyarteritis nodosa for epidemiological studies. Ann Rheum Dis. 2007;66(2):222-7.

27. Neogi T, Aletaha D, Silman AJ, Naden RL, Felson DT, Aggarwal R, et al. The 2010 American College of Rheumatology/European League Against Rheumatism classification criteria for rheumatoid arthritis: phase 2 methodological report. Arthritis Rheum. 2010;62(9):2582-91.
28. Smolen JS, Breedveld FC, Schiff MH, Kalden JR, Emery P, Eberl G, et al. A simplified disease activity index for rheumatoid arthritis for use in clinical practice. Rheumatology (Oxford). 2003;42(2):244-57.

29. Hochberg MC. Updating the American College of Rheumatology revised criteria for the classification of systemic lupus erythematosus. Arthritis Rheum. 1997;40(9):1725.

30. Gladman DD, Ibañez D, Urowitz MB. Systemic Lupus Erythematosus Disease Activity Index 2000. J Rheumatol. 2002;29(2):288-91.

31. Mukhtyar C, Lee R, Brown D, Carruthers D, Dasgupta B, Dubey S, et al. Modification and validation of the Birmingham Vasculitis Activity Score (version 3). Ann Rheum Dis. 2009;68(12):1827-32.

32. Hellmich B, Flossmann O, Gross WL, Bacon P, Cohen-Tervaert JW, Guillevin $L$, et al. EULAR recommendations for conducting clinical studies and/or clinical trials in systemic vasculitis: focus on anti-neutrophil cytoplasm antibody-associated vasculitis. Ann Rheum Dis. 2007:66(5):605-17.

33. Koyama A, Yamagata K, Makino H, Arimura Y, Wada T, Nitta K, et al. A nationwide survey of rapidly progressive glomerulonephritis in Japan: etiology, prognosis and treatment diversity. Clin Exp Nephrol. 2009;13(6): 633-50.

34. Guillevin L, Pagnoux C, Seror R, Mahr A, Mouthon L, Le Toumelin P, et al. The Five-Factor Score revisited: assessment of prognoses of systemic necrotizing vasculitides based on the French Vasculitis Study Group (FVSG) cohort. Medicine (Baltimore). 2011;90(1):19-27.

35. Takemori N, Takemori A, Ishizaki J, Hasegawa H. Enzymatic protein digestion using a dissolvable polyacrylamide gel and its application to mass spectrometry-based proteomics. J Chromatogr B Analyt Technol Biomed Life Sci. 2014;967:36-40.

36. MacLean B, Tomazela DM, Shulman N, Chambers M, Finney GL, Frewen B, et al. Skyline: an open source document editor for creating and analyzing targeted proteomics experiments. Bioinformatics. 2010;26(7):966-8.

37. Rebhan M, Chalifa-Caspi V, Prilusky J, Lancet D. GeneCards: a novel functional genomics compendium with automated data mining and query reformulation support. Bioinformatics. 1998;14(8):656-64.

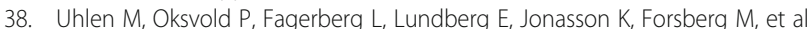
Towards a knowledge-based Human Protein Atlas. Nat Biotechnol. 2010; 28(12):1248-50.

39. Desiere F, Deutsch EW, King NL, Nesvizhskii Al, Mallick P, Eng J, et al. The PeptideAtlas project. Nucleic Acids Res. 2006;34(Database issue):D655-8.

40. Muthusamy B, Hanumanthu G, Suresh S, Rekha B, Srinivas D, Karthick L, et al. Plasma Proteome Database as a resource for proteomics research. Proteomics. 2005;5(13):3531-6.

41. Brew K, Nagase H. The tissue inhibitors of metalloproteinases (TIMPs): an ancient family with structural and functional diversity. Biochim Biophys Acta. 2010;1803(1):55-71.

42. Ries C. Cytokine functions of TIMP-1. Cell Mol Life Sci. 2014;71(4):659-72.

43. Garza MA, Wason EA, Zhang JQ. Cardiac remodeling and physical training post myocardial infarction. World J Cardiol. 2015;7(2):52-64.

44. Kim YS, Kim SH, Kang JG, Ko JH. Expression level and glycan dynamics determine the net effects of TIMP-1 on cancer progression. BMB Rep. 2012; 45(11):623-8.

45. Bjerkeli V, Halvorsen B, Damås JK, Nordøy I, Yndestad A, Aukrust $P$, et al. Expression of matrix metalloproteinases in patients with Wegener's granulomatosis. Ann Rheum Dis. 2004;63(12):1659-63.

46. Kochetov GA, Solovjeva ON. Structure and functioning mechanism of transketolase. Biochim Biophys Acta. 2014;1844(9):1608-18.

47. Mitschke L, Parthier C, Schröder-Tittmann K, Coy J, Lüdtke S, Tittmann K The crystal structure of human transketolase and new insights into its mode of action. J Biol Chem. 2010;285(41):31559-70.

48. Zhao J, Zhong CJ. A review on research progress of transketolase. Neurosci Bull. 2009;25(2):94-9.

49. Sax CM, Salamon C, Kays WT, Guo J, Yu FX, Cuthbertson RA, et al. Transketolase is a major protein in the mouse cornea. J Biol Chem. 1996; 271(52):33568-74

50. Greenlee-Wacker MC, Galvan MD, Bohlson SS. CD93: recent advances and implications in disease. Curr Drug Targets. 2012;13(3):411-20.

51. Greenlee MC, Sullivan SA, Bohlson SS. Detection and characterization of soluble CD93 released during inflammation. Inflamm Res. 2009·58(12):909-19.

52. Mälarstig A, Silveira A, Wågsäter D, Öhrvik J, Bäcklund A, Samnegård A, et al. Plasma CD93 concentration is a potential novel biomarker for coronary artery disease. J Intern Med. 2011;270(3):229-36. 
53. Youn JC, Yu HT, Jeon JW, Lee HS, Jang Y, Park YW, et al. Soluble CD93 levels in patients with acute myocardial infarction and its implication on clinical outcome. PLoS One. 2014;9(5), e96538.

54. Fonseca MI, Carpenter PM, Park M, Palmarini G, Nelson EL, Tenner AJ. ClqR(P), a myeloid cell receptor in blood, is predominantly expressed on endothelial cells in human tissue. J Leukoc Biol. 2001;70(5):793-800.

55. Pepper RJ, Hamour S, Chavele KM, Todd SK, Rasmussen N, Flint S, et al. Leukocyte and serum S100A8/S100A9 expression reflects disease activity in ANCA-associated vasculitis and glomerulonephritis. Kidney Int. 2013;83(6): $1150-8$.

56. Pruenster M, Vogl T, Roth J, Sperandio M. S100A8/A9: from basic science to clinical application. Pharmacol Ther. 2016;167:120-31.

57. Midwood KS, Orend G. The role of tenascin- $\mathrm{C}$ in tissue injury and tumorigenesis. J Cell Commun Signal. 2009;3(3-4):287-310.

58. Kaarteenaho-Wiik R, Mertaniemi P, Sajanti E, Soini Y, Pääkkö P. Tenascin is increased in epithelial lining fluid in fibrotic lung disorders. Lung. 1998; 176(6):371-80.

59. Kaarteenaho-Wiik R, Lakari E, Soini Y, Pöllänen R, Kinnula VL, Pääkkö P. Tenascin expression and distribution in pleural inflammatory and fibrotic diseases. J Histochem Cytochem. 2000;48(9):1257-68.

Submit your next manuscript to BioMed Central and we will help you at every step:

- We accept pre-submission inquiries

- Our selector tool helps you to find the most relevant journal

- We provide round the clock customer support

- Convenient online submission

- Thorough peer review

- Inclusion in PubMed and all major indexing services

- Maximum visibility for your research

Submit your manuscript at www.biomedcentral.com/submit
C) Biomed Central 